Federal Reserve Bank of Minneapolis

Research Department Staff Report 345

Revised January 2006

\title{
A Model of TFP
}

\author{
Ricardo Lagos* \\ Federal Reserve Bank of Minneapolis \\ and New York University
}

\begin{abstract}
This paper proposes an aggregative model of total factor productivity (TFP) in the spirit of Houthakker (1955-1956). It considers a frictional labor market where production units are subject to idiosyncratic shocks and jobs are created and destroyed as in Mortensen and Pissarides (1994). An aggregate production function is derived by aggregating across micro production units in equilibrium. The level of TFP is explicitly shown to depend on the underlying distribution of shocks as well as on all the characteristics of the labor market as summarized by the job-destruction decision. The model is also used to study the effects of labor-market policies on the level of measured TFP.
\end{abstract}

*I thank Gadi Barlevy, Marco Bassetto, Jeffrey Campbell, Xavier Gabaix, Nobu Kiyotaki, Erzo G. J. Luttmer, Marc Melitz, Ed Prescott, and Esteban Rossi-Hansberg for comments. Financial support from the National Science Foundation (grant SES-0232583) and the C.V. Starr Center for Applied Economics at New York University is gratefully acknowledged. The views expressed herein are those of the author and not necessarily those of the Federal Reserve Bank of Minneapolis or the Federal Reserve System. Address: Federal Reserve Bank of Minneapolis, Research Department, 90 Hennepin Avenue, Minneapolis, MN 55401, USA. E-mail: ricardo.lagos@nyu.edu. 


\section{Introduction}

This paper focuses on the theory underlying the aggregate production function and shows how labor-market policies can affect this function in general and the level of measured total factor productivity (TFP) in particular. Specifically, I construct an aggregative model of TFP in the spirit of Houthakker (1955-1956): the basic idea is to derive an aggregate production function by aggregating across active production units. In equilibrium, the levels of output, inputs, and TFP, as well as the shape of the aggregate relationship between them, depend on individual production decisions - such as which production units remain active in the face of idiosyncratic shocks - and these decisions are, in turn, affected by policies. In this way, the model can be used to study the precise interaction between all these variables explicitly.

In the model proposed here, policy affects TFP because the latter is related to the average productivity of the units that are active, and policy induces changes in the productivity composition of active units. By distorting the way in which individual production units react to

the economic environment, labor-market policies can make an economy exhibit a low level of TFP. As a result, two economies may exhibit different levels of TFP even if production units in both have access to the same technology and are subject to identical shocks.

At a theoretical level, the paper also shows that, under some conditions, a standard search model of the labor market - with its underlying meeting frictions and simple fixed-proportions micro-level production technologies - can generate an aggregate production function that looks just like the one implied by the textbook neoclassical model of growth in which firms have access to a standard constant-returns Cobb-Douglas production technology. So in this sense, from the perspective of aggregate output, inputs, and productivity, the neoclassical and the search paradigms can seem quite close. However, the search model implies a different mapping between the parameters of the aggregate production technology and observables, and this feature can be relevant for growth accounting exercises.

At a conceptual level, the paper is related to the vast literature that documents and tries 
to explain differences in TFP levels across countries. Examples include Hall and Jones (1999), Klenow and Rodríguez-Clare (1997), and Parente and Prescott (2000). In terms of explanations, this literature shares the basic idea that the level of an economy's TFP is determined by the quality of its "institutions." Hall and Jones (1999) argue that differences in observed TFP are driven by differences in the institutions and government policies they collectively refer to as "social infrastructure." Corrupt government officials, severe impediments to trade, poor contract enforcement, and government interference in production are some of their examples of bad social infrastructures that could lead to low levels of TFP.

Parente and Prescott (1994) propose that some countries have lower TFP than others because their process of technology adoption at the micro level is constrained by "barriers to riches." These barriers are essentially any institution or government policy that increases the cost of technology adoption. From that perspective, this paper can be thought of as adding labor-market policies to the list of institutions that affect the level of TFP. ${ }^{1}$

The paper is also related to the large body of work that tries to account for differences in macroeconomic performance, usually employment rates and labor productivity, with differences in labor-market policies, typically unemployment benefits or employment protection. Hopenhayn and Rogerson (1993), Marimon and Zilibotti (1999), Millard and Mortensen (1997), Restuccia and Rogerson (2004), and Veracierto (2001) are some examples of this line of work.

The rest of the paper is organized as follows. Section 2 lays out the model. The equilibrium is characterized in Section 3. In Section 4, an aggregation result similar in spirit to the classic contribution of Houthakker (1955-1956) is derived for a canonical dynamic equilibrium search economy. This section also shows how, when aggregate inputs are measured appropri-

\footnotetext{
${ }^{1}$ Unlike Hall and Jones (1999), Parente and Prescott (2000), or Acemoglu and Zilibotti (2001), this paper is not about development economics, in the sense that it does not attempt to explain why some countries are 30 times richer than others in per-capita terms. The emphasis on labor-market institutions makes the analysis more relevant to study productivity differences among a relatively homogeneous set of countries (or sectors). For example, Hall and Jones (1999) and Klenow and Rodríguez-Clare (1997) report productivity differences among OECD countries, with France and Italy having a higher level of TFP and Germany a much lower level relative to the United States. These observations may seem striking, especially given the conventional wisdom that France and Italy have distorted labor markets vis-à-vis the United States. These are examples of the types of questions that the model developed here is well suited to address.
} 
ately, the level of TFP depends on all the characteristics of the labor market summarized by the job-destruction decisions. Section 5 introduces four policies - employment subsidies, hiring subsidies, firing taxes, and unemployment benefits - and studies their effects on TFP. Section 6 puts the theoretical results of the previous sections into perspective. Section 6.1 explains the similarities and differences between the aggregation result obtained in Section 4 and Houthakker's contribution, and relates the general approach to the literature on aggregation of production functions that dates back to the late 1940's. Since empirically, the level of TFP is measured as a residual, it depends critically on the measurement of the inputs of production. Section 6.2 discusses some of these measurement issues and uses the theory to study their implications for the nature of the TFP residual. Section 6.3 draws some connections to the standard growth accounting exercises. In Section 7, a calibrated version of the model is used to quantify the effects of labor-market policies on the level of TFP. Section 8 extends the basic model to allow for serially correlated shocks and state-dependent destruction rates, and it generalizes the main aggregation result. Section 9 concludes. All propositions are proven in Appendix A. Appendix B contains additional extensions and some auxiliary results.

\section{The Model}

The labor market is modeled as in Mortensen and Pissarides (1994). ${ }^{2}$ Time is continuous and the horizon infinite. There is a continuum of infinitely lived agents of two types: workers and firms. Both types are risk-neutral. The size of the labor force is normalized to unity, whereas the number of firms will be determined endogenously by free entry. Workers derive utility from consumption and - in one of the specifications developed below - also suffer disutility from working.

\footnotetext{
${ }^{2}$ There are at least three reasons for carrying out the analysis in a search and matching framework. First, as will be discussed in Section 5, the labor-market policies considered will have testable implications not only for the level of TFP but also for the unemployment rate and the job-creation and job-destruction rates. Second, an explicit treatment of unemployment is relevant because - as will be shown in Sections 6 and 7 - the unemployment rate will affect empirical measures of TFP for the ways of measuring aggregate inputs that can be found in the literature. And finally, this framework has already been used extensively to analyze the effects of similar policies on many other aggregate labor-market outcomes (see Ljungqvist and Sargent, 2000, Pissarides, 2000, and references therein).
} 
Each firm has a single job that can be either filled or vacant and searching. Similarly, workers can be either employed by a firm or unemployed and searching. No new offers arrive while an agent is in a relationship. I abstract from capital accumulation and assume an exogenous rental rate of capital, $c$. The aggregate stock of capital, $K$, will be determined by demand.$^{3}$

Assume meeting frictions can be represented by a function $m(u, v)$ that determines the instantaneous number of meetings as a function of the numbers of searchers on each side of the market, namely, unemployed workers $u$ and vacancies $v$. Suppose $m$ exhibits constant returns to scale and is increasing in both arguments. Let $q(\theta)$ denote the (Poisson) rate at which a vacancy contacts an unemployed worker, with $\theta=v / u{ }^{4}$

Each firm has access to a technology $f(x, n, k)$ that combines the hours supplied by the worker it employs, $n$, and capital, $k$, to produce a homogeneous consumption good. The matchspecific productivity level is stochastic and indexed by the random variable $x$. I assume that

$$
f(x, n, k)=x \min (n, k)
$$

and interpret $k$ as the firm's capacity or scale of operation. Thus, output is linear in hours but is bounded above by the stock of capital the firm is operating with. The convention is that the technology is such that all projects have the same scale of operation $k$. Every firm has to rent and put in place $k$ units of capital to be able to engage in search while vacant and to produce while filled. ${ }^{5}$ This captures the notion that hours are a fully flexible factor but capital is relatively fixed. Firms rent capital from a competitive market at flow cost $c$.

The process that changes the match-specific productivity, $x$, is Poisson with finite arrival rate $\lambda$. When a match of productivity $x$ suffers a change, the new value $x^{\prime}$ is a draw from

\footnotetext{
${ }^{3}$ This is the usual small, open economy assumption. The model abstracts from saving and accumulation because the focus here is on isolating the effects of labor-market policies on the level of TFP. But even in the context of trying to explain income differences, Prescott (1998) and Parente and Prescott (2000) conclude that one cannot rely on policies that cause differences in saving rates, as they do not vary systematically with countries' incomes.

${ }^{4}$ Note that $q(\theta)=m(1 / \theta, 1)$ and hence $q^{\prime}<0$. The rate at which a worker contacts a vacancy is $\theta q(\theta)$ and is increasing in $\theta$. For some of the existence proofs, assuming $\lim _{\theta \rightarrow 0} q(\theta)=\infty$ will also be useful.

${ }^{5}$ The idea is that in order to search, the firm must have borrowed some capital, say, to set up a plant, and plants come in a single size, $k$.
} 
the fixed distribution $G(\cdot)$. So the productivity process is persistent (since $\lambda<\infty$ ), butconditional on change - it is independent of the firm's previous state. ${ }^{6}$ The Poisson process and the productivity draws are independently and identically distributed across firms, and there is no aggregate uncertainty. The focus will be on steady-state outcomes.

In the next section, I will show that there is a unique productivity level $R_{t}$ such that active matches dissolve if productivity ever falls below that level and new matches form only if their initial productivity is at least $R_{t} \cdot{ }^{7}$ Let $H_{t}(x)$ denote the cross-sectional productivity distribution of active matches. That is, $H_{t}(x)$ is the fraction of matches producing at productivities $x$ or lower at time $t$. The time path of $\left(1-u_{t}\right) H_{t}(x)$, the mass of matches producing at productivities $x$ or lower at time $t$, is given by $^{8}$

$$
\begin{aligned}
\frac{d}{d t}\left[\left(1-u_{t}\right) H_{t}(x)\right]= & \lambda\left(1-u_{t}\right)\left[1-H_{t}(x)\right]\left[G(x)-G\left(R_{t}\right)\right]+\theta q(\theta) u_{t}\left[G(x)-G\left(R_{t}\right)\right] \\
& -\lambda\left(1-u_{t}\right) H_{t}(x) G\left(R_{t}\right)-\lambda\left(1-u_{t}\right) H_{t}(x)[1-G(x)] \\
& -\delta\left(1-u_{t}\right) H_{t}(x)
\end{aligned}
$$

The first term accounts for the matches with productivities above $x$ that get innovations below $x$ but above $R_{t}$. The newly formed matches that start off with productivities no larger than $x$ are in the second term. The third term represents the matches with productivity in the interval $\left[R_{t}, x\right]$ that get shocks below $R_{t}$ and are destroyed. The fourth term accounts for those matches in the same interval that "move up" by virtue of having drawn productivities larger than $x$. The parameter $\delta<\infty$ denotes the arrival rate of an independent Poisson process that causes separations for reasons unrelated to the match-specific productivity shocks. The last

\footnotetext{
${ }^{6}$ This is the process used by Mortensen and Pissarides (1994). For reasons that will become clear shortly, Section 8 generalizes the model by specifying that when a match of productivity $x$ suffers a change, the new value $x^{\prime}$ is a draw from the fixed distribution $G(\cdot \mid x)$. If $G\left(\cdot \mid x_{1}\right)<G\left(\cdot \mid x_{0}\right)$ when $x_{0}<x_{1}$, then apart from being persistent, the idiosyncratic shocks are also positively correlated over time.

${ }^{7}$ Mortensen and Pissarides (1994) work with a bounded support and assume new matches start off with the highest productivity. I relax these assumptions and treat active and new matches symmetrically. In the model considered here, the initial productivity of a match is a nondegenerate random variable drawn from the same distribution as the innovations to active matches.

${ }^{8}$ The fact that active matches will form and continue only for productivities at least as large as $R_{t}$ means that $H_{t}\left(R_{t}\right)=0$. Thus, the derivation focuses on $x \geq R_{t}$.
} 
term accounts for the matches in the interval $\left[R_{t}, x\right]$ that are destroyed for exogenous reasons. ${ }^{9}$ In steady state,

$$
\begin{aligned}
H(x) & =\left[\frac{\lambda}{\delta+\lambda}+\frac{\theta q(\theta) u}{(\delta+\lambda)(1-u)}\right][G(x)-G(R)] \\
u & =\frac{\delta+\lambda G(R)}{\delta+\lambda G(R)+\theta q(\theta)[1-G(R)]} .
\end{aligned}
$$

Using (2), the steady-state cross-sectional productivity distribution becomes

$$
H(x)=\frac{G(x)-G(R)}{1-G(R)} .
$$

Next, I consider the problems faced by a worker and a firm. The values of unemployment and employment to a worker are denoted $U$ and $W(x)$, respectively, and solve

$$
\begin{aligned}
r U & =b+\theta q(\theta) \int \max [W(z)-U, 0] d G(z) \\
r W(x) & =w(x)+\lambda \int \max [W(z)-U, 0] d G(z)-(\delta+\lambda)[W(x)-U],
\end{aligned}
$$

where $r$ is the discount rate, $b \geq 0$ denotes a worker's flow income while unemployed, and $w(x)$ is the wage earned by a worker employed in a match of productivity $x .^{10}$

Firms can be either filled or vacant and searching. The problem of a searching firm is summarized by

$$
r V=-c k+q(\theta) \int \max [J(z)-V, 0] d G(z),
$$

where $V$ is the asset value of a vacancy and $J(x)$ the asset value of a filled job with productivity $x$. Letting $\pi(x)$ denote flow profit, $J(x)$ satisfies

$$
r J(x)=\pi(x)+\lambda \int \max [J(z)-V, 0] d G(z)-(\delta+\lambda)[J(x)-V],
$$

where $\pi(x)=x \min (n, k)-w(x)-c k-\phi n-C(x, \phi) k$. Instantaneous profit is the residual output after the wage $w(x)$ and all other costs of production have been paid out. This formulation has three such costs: the rental on capital, $c k$; a variable cost, $\phi n$, that can be managed by

\footnotetext{
${ }^{9}$ In Section 8 I extend the analysis to the case in which the rate $\delta$ is a decreasing function of the idiosyncratic productivity parameter $x$. I will discuss the reasons why this extension may be worth exploring shortly.

${ }^{10}$ This formulation assumes the worker suffers no disutility from work; but see Appendix B.
} 
varying hours; and a fixed cost $C$ per unit of capital. ${ }^{11}$ The variable cost $\phi n$ and the fixed cost $C k$ are introduced to allow for the possibility of labor hoarding and underutilization of capital, two pervasive features of the data. In the presence of these costs, for some parametrizations it will be possible that at low productivity realizations, the firm may choose to keep the worker employed despite requiring that she supply zero hours. In Sections 4 and 6.2 I will show that this type of labor hoarding has interesting aggregate implications when it occurs in equilibrium.

To fix ideas, one can think of $\phi$ as the cost of electricity, for instance, with electricity usage being proportional to hours worked. Alternatively, in Appendix B I show how to modify (5) and $\pi(x)$ to get an equivalent formulation in which $\phi n$ is the worker's disutility from supplying $n$ hours to her employer. Either way, the key observation is that $\phi>0$ is necessary for the model to display the type of labor hoarding described above. Intuitively, if the marginal product of labor, $x$, is lower than its marginal cost, $\phi$ (expressed either in terms of resources to the firm under the first interpretation or in terms of disutility to the worker under the second), then efficiency will require the match to set $n=0$ : the worker should not supply hours to the firm. Under these circumstances, whether the firm and the worker should choose to preserve the match is a different matter. One possibility is that the instances in which the firm-worker pair sets $n=0$ are also the cases in which the pair chooses to dissolve the match. Alternatively, one could imagine that the pair may choose $n=0$, yet decide to preserve the match anticipating that the low realization of the idiosyncratic shock may be reversed soon. In the first scenario no hoarding is exhibited. In the latter it is. The fixed cost $C$ introduced in this section makes it possible for the second scenario to arise in an equilibrium with endogenous separations.

Notice that if $C=\phi=0$, then the model reduces to the standard setup of Mortensen and Pissarides (1994). For this special case, all the results on how labor-market policies affect the level of TFP still apply even though the equilibrium does not exhibit hoarding. In this sense, neither of these costs is essential. However, in the presence of the variable cost $\phi$ and the fixed

\footnotetext{
${ }^{11}$ The cost $C$ is fixed in the sense that the firm can avoid it only by shutting down, but-as the notation indicates - it may depend on the realization of the idiosyncratic shock and possibly also on the parameter $\phi$. More on this below.
} 
$\operatorname{cost} C$, the model is also able to deliver equilibrium hoarding. And as it turns out, this feature has interesting implications for how the aggregate production function looks.

Since the fixed $\operatorname{cost} C$ is perhaps the only nonstandard element of the model, several remarks are in order. First, at a technical level, incorporating a fixed-cost specification that is decreasing in the shock $x$ is introduced in this section as a simple device to avoid a "flat spot" in flow profit that would otherwise carry over to the value functions. ${ }^{12}$ Second, in Section 8 I drop the fixed cost and redo the analysis in a version of the model in which the draws of the idiosyncratic shock are correlated over time. In another extension explored in Section 8, I again drop the fixed cost and show that the key insights also apply in a version of the model in which the destruction rate $\delta$ is decreasing in the productivity shock. I wish to emphasize that these alternative specifications seem natural (perhaps even more so than the usual benchmark in which the shocks hitting the match are independent of its state) and are able to deliver hoarding without the fixed cost $C .{ }^{13}$ Given all this, and for expositional purposes, I will - for the time being - use a particularly convenient specification for the fixed cost, namely, $C(x, \phi)=\max (\phi-x, 0){ }^{14}$

\section{Equilibrium}

I follow the bulk of the search literature by letting $\beta \in[0,1)$ and assuming the instantaneous wage $w(x)$ and labor supply $n$ solve $\max [W(x)-U]^{\beta}[J(x)-V]^{1-\beta}$ at all times. The optimal choice of hours is

$$
n(x)= \begin{cases}k & \text { if } \phi<x \\ 0 & \text { if } x \leq \phi\end{cases}
$$

\footnotetext{
${ }^{12}$ See Appendix B, in particular the discussion around equation (47), for details.

${ }^{13}$ Flow profit has a flat spot in both of these alternative formulations, but the serially correlated shocks ensure that the value functions do not inherit this flat spot.

${ }^{14}$ This formulation is convenient because it will imply that the flow profit is affine and strictly increasing in $x$. Since - even accepting the presence of a fixed cost - this particular formulation may seem somewhat contrived, in Appendix B I redo the whole analysis with a more general specification for the fixed cost and show that $\frac{\partial C(x, \phi)}{\partial x}<0$ is all that is needed. But again, see Section 8 for alternative specifications that do not rely on firms having to bear any fixed cost $C$.
} 
and substituting it into the flow profit function gives $\pi(x)=(x-\phi-c) k-w(x)$ for all $x$. The first-order condition for the instantaneous wage, $w(x)$, is

$$
(1-\beta)[W(x)-U]=\beta[J(x)-V]
$$

Letting $S(x)=J(x)+W(x)-U-V$ denote the surplus from a match, notice that (9) implies $J(x)=(1-\beta) S(x)$ and $W(x)-U=\beta S(x)$. These, together with (4), (5), and (7), imply

$$
(r+\delta+\lambda) S(x)=(x-\phi-c) k-r U+\lambda \int \max [S(z), 0] d G(z),
$$

where

$$
r U=b+\frac{\beta}{1-\beta} k c \theta .
$$

In deriving (10) I have already acknowledged that free entry of firms will cause $r V=0$ in equilibrium. Since $S^{\prime}(x)=\frac{k}{r+\delta+\lambda}>0$, there exists a unique $R$ such that $S(x)>0$ iff $x>R$. Hence a firm-worker pair destroys an existing match (and chooses not to form a new match) if it draws a productivity $x<R$. With this reservation strategy, the surplus can be written as

$$
(r+\delta+\lambda) S(x)=(x-\phi-c) k-r U+\lambda \int_{R} S(z) d G(z)
$$

The value functions and (9) can be combined to obtain the instantaneous wages and profit:

$$
\begin{aligned}
& w(x)=\beta(x-\phi-c) k+(1-\beta) r U \\
& \pi(x)=(1-\beta)[(x-\phi-c) k-r U] .
\end{aligned}
$$

Intuitively, the wage is a weighted average of output (net of the rental on capital and the variable and fixed costs) and the worker's reservation wage.

Next, I characterize the job-creation and job-destruction decisions as summarized by $\theta$ and $R$, respectively. Evaluating (11) at $x=R$ yields $\lambda \int_{R} S(z) d G(z)=r U-(R-\phi-c) k$. Notice that since the expected capital gain on the left side is positive, at $x=R$ net output is smaller than the worker's reservation wage. Thus, (12) and (13) imply that $w(R)<r U$ and $\pi(R)<0$ : workers and firms sometimes tolerate instantaneous payoffs below those they could get by 
separating, in anticipation of future productivity improvements. ${ }^{15}$ Substituting this simpler expression for the expected capital gain term into (11) gives

$$
S(x)=\frac{x-R}{r+\delta+\lambda} k .
$$

Evaluating (11) at $x=R$ and using (14) to substitute $S(\cdot)$ yields what is usually referred to as the job-destruction condition:

$$
R-\phi-c-\left(\frac{b}{k}+\frac{\beta}{1-\beta} c \theta\right)+\frac{\lambda}{r+\delta+\lambda} \int_{R}(x-R) d G(x)=0 .
$$

As is standard, the destruction decision is independent of scale if $b$ is. The natural interpretation of $b$ is that it is unemployment insurance income. Along these lines, if one lets $b=\tau_{b} E_{G}[w(x) \mid x \geq R]$, where $\tau_{b} \in[0,1)$ is the replacement rate, then $b=\hat{b} k$, with

$$
\hat{b}=\frac{\tau_{b} \beta[\tilde{x}(R)-\phi-c+c \theta]}{1-(1-\beta) \tau_{b}}
$$

and $\tilde{x}(R) \equiv E_{G}[x \mid x \geq R]=[1-G(R)]^{-1} \int_{R} x d G(x)$. Under this specification, $b$ is linear in $k$ so (15) is independent of $k$ and becomes

$$
R-\frac{\tau_{b} \beta \tilde{x}(R)}{1-(1-\beta) \tau_{b}}-\frac{\left(1-\tau_{b}\right)(\phi+c)}{1-(1-\beta) \tau_{b}}-\frac{\beta c \theta}{(1-\beta)\left[1-(1-\beta) \tau_{b}\right]}+\frac{\lambda}{r+\delta+\lambda} \int_{R}(x-R) d G(x)=0 .
$$

In what follows I will always abstract from scale effects caused by unemployment income $b$ by assuming it is a fraction of the average going wage.

Substituting the equilibrium condition $r V=0$ in (6) implies $(1-\beta) \int_{R} S(x) d G(x)=\frac{c k}{q(\theta)}$. That is, the expected profit from a filled job equals the expected hiring cost in an equilibrium with free entry. Using (14) to substitute $S(\cdot)$ out of this expression yields what is often referred to as the job-creation condition:

$$
\frac{1-\beta}{r+\delta+\lambda} \int_{R}(x-R) d G(x)-\frac{c}{q(\theta)}=0 .
$$

\footnotetext{
${ }^{15}$ This feature of the model is a consequence of the costly and time-consuming meeting process, as noted by Mortensen and Pissarides (1994).
} 
The job-creation and job-destruction conditions jointly determine $R$ and $\theta$, and under the maintained assumptions, they are independent of scale, $k{ }^{16}$ For given $c$ and $\phi$, an equilibrium is a vector $[\theta, R, H, U, w, u, K]$ such that $(\theta, R)$ jointly solve (15) and (16); given $(\theta, R), H$ satisfies (3); $U$ is given by (10); $w$ is given by (12); and $u$ is given by (2). In addition, the market for capital services should clear, so the aggregate supply of capital $K$ must satisfy $K=[1-(1-\theta) u] k$, where the right side is the total demand for capital (coming from both matched and unmatched firms). Note that in parametrizations that result in $R<\phi$, the capital and workers in matches with realizations in $[R, \phi)$ remain employed but are not engaged in production. The firms in these states have excess capacity and hoard labor. The following section provides a sharper characterization of aggregate outcomes for a particular distribution of idiosyncratic shocks.

\section{Aggregation}

Let $K_{e}$ denote the capital in place at all the firms with filled jobs, i.e.,

$$
K_{e}=\frac{1-u}{1-(1-\theta) u} K
$$

Aggregate output, $Y$, and the total number of hours worked, $N$, are given by

$$
Y=(1-u) \int_{\mu} f[x, n(x), k] d H(x),
$$

and $N=(1-u) \int_{\mu} n(x) d H(x)$, with $\mu \equiv \max (R, \phi)$. Using (1) and (8) gives

$$
\begin{aligned}
Y\left(K_{e}, \mu\right) & =[1-H(\mu)] K_{e} E_{H}(x \mid x \geq \mu) \\
N & =[1-H(\mu)] K_{e}
\end{aligned}
$$

where $E_{H}(x \mid x \geq \mu)=[1-H(\mu)]^{-1} \int_{\mu} x d H(x)$. Intuitively, since every firm-worker pair is setting hours either to zero or to full capacity $k$, the aggregate number of hours worked is just

\footnotetext{
${ }^{16}$ See Lemma 1 in Appendix B for conditions under which the pair $(\theta, R)$ that solves $(15)$ and $(16)$ exists and is unique.
} 
equal to the fraction of firm-worker pairs who engage in production times the total capital stock in filled jobs. Similarly, aggregate output equals the number of active units of capital, $[1-H(\mu)] K_{e}$, weighted by their average productivity. ${ }^{17}$ Following Houthakker (1955-1956), one could imagine solving (19) for the "aggregate labor demand" by active firms, $\mu\left(K_{e}, N\right)$, and then substituting it in (18) to obtain $Y\left[K_{e}, \mu\left(K_{e}, N\right)\right]$. Hereafter, I use $F\left(K_{e}, N\right)$ to denote $Y\left[K_{e}, \mu\left(K_{e}, N\right)\right]$ to simplify notation and to stress the fact that this is the economy's "aggregate production function." This aggregate production function exhibits constant returns to scale even for an arbitrary $H$. To see this, notice that $\mu\left(K_{e}, N\right)$ is homogeneous of degree zero, and hence (18) indicates that for any $\zeta>0, F\left(\zeta K_{e}, \zeta N\right)=\zeta F\left(K_{e}, N\right) .{ }^{18}$

Suppose idiosyncratic shocks are draws from a Pareto distribution:

$$
G(x)= \begin{cases}0 & \text { if } x<\varepsilon \\ 1-\left(\frac{\varepsilon}{x}\right)^{\alpha} & \text { if } \varepsilon \leq x\end{cases}
$$

where $\varepsilon>0$ and $\alpha>1$. Then, provided $R \geq \varepsilon, 1-G(R)=\left(\frac{\varepsilon}{R}\right)^{\alpha}$ and hence $G(x)-G(R)=$ $(\varepsilon / R)^{\alpha}\left[1-(R / x)^{\alpha}\right]$ for any $x \geq R$. Substituting these expressions in (3) yields

$$
H(x)= \begin{cases}0 & \text { if } x<R \\ 1-\left(\frac{R}{x}\right)^{\alpha} & \text { if } R \leq x .\end{cases}
$$

So the steady-state productivity distribution of active matches follows a Pareto distribution with parameters $R$ and $\alpha$. Using $(21), 1-H(\mu)=\left(\frac{R}{\mu}\right)^{\alpha}$ and $E_{H}(x \mid x \geq \mu)=\frac{\alpha}{\alpha-1} \mu$, so the aggregates (18) and (19) specialize to $Y\left(K_{e}, \mu\right)=\frac{\alpha}{\alpha-1} R^{\alpha} \mu^{1-\alpha} K_{e}$ and $N=(R / \mu)^{\alpha} K_{e}$. Inverting the latter yields $\mu=\left(K_{e} / N\right)^{1 / \alpha} R$, and substituting this in the former implies

$$
F\left(K_{e}, N\right)=A K_{e}^{\gamma} N^{1-\gamma},
$$

\footnotetext{
${ }^{17}$ As mentioned in footnote 7, Mortensen and Pissarides (1994) assume that $G$ has support $[0,1]$ and that all new matches start off with productivity 1 . So, with $\delta=0$, aggregate output in their model evolves according to

$$
\dot{Y}=\theta q(\theta) u k-\lambda Y+\lambda(1-u) k \int_{\mu}^{1} x d G(x) .
$$

Replacing $(1-u) k$ with $K_{e}$, steady-state output is $Y=\frac{\theta q(\theta) u k}{\lambda}+[1-H(\mu)] K_{e} E_{H}(x \mid x \geq \mu)$, which looks like (18) except for the first term. Assuming that the initial productivity of a new match is a random draw from $G-$ just as the innovations to the productivity of ongoing matches - allows for a density $G$ with unbounded support. In addition, this alternative assumption smooths aggregate output by getting rid of the "spike" $\theta q(\theta) u k \lambda^{-1}$.

${ }^{18}$ Also, from (18), $F_{2}\left(K_{e}, N\right)=-\mu_{2}\left(K_{e}, N\right) K_{e} \mu d H(\mu)$, and from $(19),-\mu_{2}\left(K_{e}, N\right) K_{e} d H(\mu)=1$. Thus, $F_{2}\left(K_{e}, N\right)=\mu$. So the marginal product of labor in the aggregate production function is equal to the marginal product of the least efficient unit of labor employed in production. I owe this argument to Erzo G. J. Luttmer.
} 
where

$$
A=\frac{R}{1-\gamma}
$$

and $\gamma \equiv 1 / \alpha$. This aggregation result is reminiscent of the classic aggregation result of Houthakker (1955-1956). ${ }^{19}$ The factor $A$ is what macroeconomists normally refer to as TFP. Its level depends on $\alpha$, a parameter of the primitive distribution of productivity shocks, as well as on all the characteristics of the labor market as summarized by the destruction decision, $R$.

\section{Labor-Market Policies and the Level of TFP}

This section considers the effects of four policies: employment subsidies, hiring subsidies, firing taxes, and unemployment benefits. I follow Pissarides (2000) and model the subsidies as transfers from the government to the firm and the firing taxes as payments from the firm to the government. ${ }^{20}$ The value function $W(x)$ is still given by (5), while (4), (6), and (7) now generalize to

$$
\begin{aligned}
r U & =\tau_{b}+\theta q(\theta) \int \max \left[W_{o}(z)-U, 0\right] d G(z) \\
r V & =-c k+q(\theta) \int \max \left[J_{o}(z)+\tau_{h} k-V, 0\right] d G(z) \\
r J(x) & =\pi(x)+\tau_{e} k+\lambda \int \max \left[J(z)-V+\tau_{f} k, 0\right] d G(z)-(\delta+\lambda)\left[J(x)-V+\tau_{f} k\right] .
\end{aligned}
$$

The policy variables are a hiring subsidy, $\tau_{h}$, an employment subsidy, $\tau_{e}$, a firing tax, $\tau_{f}$, and unemployment benefits, $\tau_{b} .{ }^{21}$ In Appendix B I show that if $G$ is as in (20), the job-destruction

\footnotetext{
${ }^{19} \mathrm{I}$ discuss this relationship at some length in Section 6.

${ }^{20} \mathrm{I}$ assume that upon separation, the firm must pay the firing tax to the government because in the present setup, firing taxes would be completely neutral under the alternative scheme in which the firm compensates the fired worker directly.

${ }^{21}$ All payments are assumed to be proportional to the firm's size, as measured by $k$. For example, upon separation, the firm is taxed $\tau_{f} k$, and while unemployed, the worker receives $\tau_{b} k$ from the government. This assumption, borrowed from Pissarides (2000), is convenient because it ensures that policies introduce no scale effects into the job-creation and job-destruction decisions. Also, to keep the analysis simple, I will ignore the government's financing constraints. A natural extension would be requiring the government to run a balanced budget. A simple example of a scheme that is self-financing in the steady state is $\tau_{f}=\tau_{h}$ and $\tau_{b}=\tau_{e}=0$.
} 
and job-creation conditions that determine the equilibrium pair $(\theta, R)$ are

$$
\begin{aligned}
R-\phi-c+\tau_{e}+r \tau_{f}-\left(\tau_{b}+\frac{\beta}{1-\beta} c \theta\right)+\frac{\lambda \varepsilon^{\alpha} R^{1-\alpha}}{(\alpha-1)(r+\delta+\lambda)} & =0 \\
\frac{\varepsilon^{\alpha} R^{1-\alpha}}{(\alpha-1)(r+\delta+\lambda)}+\left(\frac{\varepsilon}{R}\right)^{\alpha}\left(\tau_{h}-\tau_{f}\right)-\frac{c}{(1-\beta) q(\theta)} & =0 .
\end{aligned}
$$

The main properties of the equilibrium are summarized as follows.

Proposition 1 Suppose $\varepsilon+(\alpha-1)(r+\delta+\lambda)\left(\tau_{h}-\tau_{f}\right)>0$. Let $\theta_{\epsilon}^{*}$ be defined by $q\left(\theta_{\epsilon}^{*}\right)=$ $\frac{(\alpha-1)(r+\delta+\lambda) c}{(1-\beta)\left[\varepsilon+(\alpha-1)(r+\delta+\lambda)\left(\tau_{h}-\tau_{f}\right)\right]}$ and $\phi_{\epsilon}=\left[1+\frac{\lambda}{(\alpha-1)(r+\delta+\lambda)}\right] \varepsilon-\left(1+\frac{\beta}{1-\beta} \theta_{\epsilon}^{*}\right) c+\tau_{h}+r \tau_{f}-\tau_{b}$. If $\varepsilon+\alpha(r+\delta+\lambda)\left(\tau_{h}-\tau_{f}\right)>0$, then for any $\phi>\phi_{\varepsilon}$ (a) there exists a unique equilibrium, (b) $R>\varepsilon$, (c) $\partial R / \partial \phi>0$ and (d) $\partial \theta / \partial \phi<0$. If in addition $\phi_{\epsilon}-\varepsilon>0$, then (e) there is a nondegenerate interval $\left(\phi_{\epsilon}, \widetilde{\phi}\right)$ such that $R(\phi)<\phi$ for all $\phi \in\left(\phi_{\epsilon}, \widetilde{\phi}\right)$.

Aggregate output is still given by (22); the aggregate stock of capital in filled jobs, $K_{e}$, is still given by (17); and the aggregate number of hours worked, $N$, is still given by $N=(R / \mu)^{\alpha} K_{e}$. In addition, if the measure of capital used to construct aggregate output is $K_{e}$, then the level of TFP is still as in (23). The following result, which holds under the assumptions stated in Proposition 1, summarizes the effects that labor-market policies have on the level of TFP.

Proposition 2 Employment subsidies and firing taxes reduce A. Hiring subsidies and unemployment benefits increase $A$.

Since $A$ is proportional to $R$, policies have the same qualitative effect on TFP as they have on the destruction rate. Proposition 2 is illustrated in Figure 1.

Employment subsidies make firms more tolerant of low productivity realizations and hence lower the average productivity of active firms. All else equal, an economy with relatively high subsidies to continued employment will exhibit a low job-destruction rate, a high jobcreation rate, and hence low levels of unemployment and measured TFP. Firing taxes have a similar qualitative effect on job destruction, but that mechanism is reinforced by a relatively low rate of job creation (which reduces the reservation wage and hence makes firms even more 


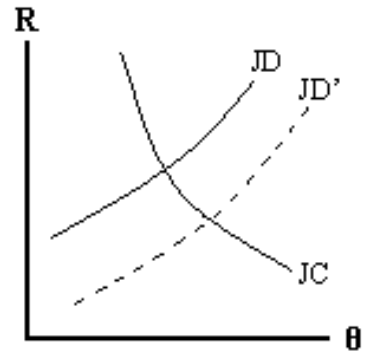

(a) Employment Subsidy

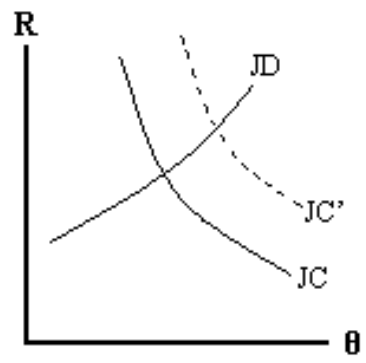

(c) Hiring Subsidy

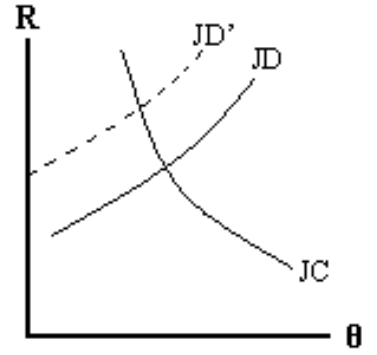

(b) Unemployment Benefits

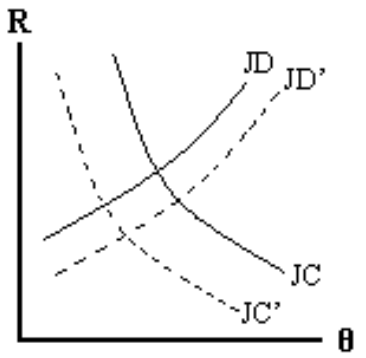

(d) Firing Tax

Figure 1: Equilibrium effects of policies on job-creation (JC) and job-destruction (JD) rates

tolerant of low productivity realizations). So firing restrictions will reduce measured TFP, as well as the job-creation and job-destruction rates. Hiring subsidies have no direct effect on the destruction decision, but they do stimulate job creation. This increases market tightness, which in turn increases the worker's outside option and raises measured TFP, job creation, and job destruction. Unemployment benefits also cause $R$ to rise through an increase in the worker's reservation wage. Consequently, economies with relatively high unemployment benefits will tend to exhibit relatively high levels of TFP and unemployment. ${ }^{22}$

\footnotetext{
${ }^{22}$ Notice that these results obtain if factor inputs are measured rather accurately, so that the TFP residual corresponds to (23). But naturally, what is measured as the TFP residual, as well as how it responds to policy, will depend on the accuracy with which factor inputs are measured. See Sections 6.2 and 7 below.
} 


\section{Discussion}

In this section I elaborate on several issues that were raised in the previous sections. First, I explain the similarities and differences between the well-known aggregation result of Houthakker (1955-1956) and the one I obtained in Section 4. I then use the aggregation result to show how the observed level of TFP depends on the measurement of factor inputs. Finally, I comment on the implications of the theory for the standard growth accounting exercises that presume frictionless markets and competitive pricing.

\subsection{Houthakker}

Houthakker (1955-1956) studies the problem of aggregating the technological possibilities of individual production units (e.g., firms) into a production function for a group of units (e.g., an industry). He considers a set of heterogeneous production units that can employ two variable factors, $x_{1}$ and $x_{2}$, to produce output, $x_{0}$, and face capacity constraints due to a fixed, unmod-

eled factor. Each unit $j$ has access to a fixed-proportions technology $x_{0 j}=\min \left(\frac{x_{1}}{a_{1 j}}, \frac{x_{2}}{a_{2 j}}, \varphi_{j}\right)$. That is, $a_{1 j}$ units of input 1 and $a_{2 j}$ units of input 2 are needed to produce one unit of $x_{0}$, and the unmodeled fixed inputs limit the production unit's possible output to $\varphi_{j}$ units of $x_{0}$. The setup is static and the notion of equilibrium partial: each production unit $j$ seeks to maximize profit, $1-x_{1 j} p_{1}-x_{2 j} p_{2}$, where $p_{i}$ is the price of input $i=1,2$ (the price of output has been normalized to 1). The optimal choice of inputs for production unit $j$ is

$$
x_{i j}= \begin{cases}a_{i j} \varphi_{j} & \text { if } 1-a_{1 j} p_{1}-a_{2 j} p_{2} \geq 0 \\ 0 & \text { if } 1-a_{1 j} p_{1}-a_{2 j} p_{2}<0\end{cases}
$$

for $i=1,2$. Units that can attain positive profit produce at full capacity, i.e., they set $x_{0 j}=\varphi_{j}$, whereas those that cannot, remain inactive, i.e., they set $x_{i j}=0$ for $i=0,1,2$. Let $a=\left(a_{1}, a_{2}\right)$ and assume a density, $\varphi(a)$, of (the various levels of) capacity constraints across production cells. Observe that total output is

$$
X_{0}=f_{0}\left(p_{1}, p_{2}\right)
$$


where $f_{0}\left(p_{1}, p_{2}\right) \equiv \int_{0}^{p_{1}^{-1}} \int_{0}^{\frac{1+a_{1} p_{1}}{p_{2}}} \varphi(a) d a$. The total amount used of variable input $i$ is

$$
X_{i}=f_{i}\left(p_{1}, p_{2}\right) \text { for } i=1,2,
$$

where $f_{i}\left(p_{1}, p_{2}\right) \equiv \int_{0}^{p_{1}^{-1}} \int_{0}^{\frac{1+a_{1} p_{1}}{p_{2}}} a_{i} \varphi(a) d a$. Then, similar to what was done in Section 4 , one may be able to solve the two expressions in (28) for prices, i.e., $p_{i}=g_{i}\left(X_{1}, X_{2}\right)$, and then substitute these expressions back into (27) to get a functional relationship between aggregate output and the aggregate quantities of the variable inputs: the aggregate production function. Specifically, $X_{0}=F\left(X_{1}, X_{2}\right)$, where $F\left(X_{1}, X_{2}\right) \equiv f_{0}\left[g_{1}\left(X_{1}, X_{2}\right), g_{2}\left(X_{1}, X_{2}\right)\right]$. Clearly, the shape of the aggregate $F$ will depend on the particular shape of the distribution of capacities, $\varphi$. Houthakker's key insight was that if $\varphi$ is of the "generalized Pareto type," i.e., $\varphi(a)=$ $\kappa a_{1}^{\alpha_{1}-1} a_{2}^{\alpha_{2}-1}$, with $\alpha_{1} \geq 1, \alpha_{2} \geq 1$, and $\kappa \geq 0$, then $F\left(X_{1}, X_{2}\right)=C X_{1}^{\gamma_{1}} X_{2}^{\gamma_{2}}$, where $C, \gamma_{1}$, and $\gamma_{2}$ are expressions involving only the parameters of the primitive distribution $\left(\alpha_{1}, \alpha_{2}\right.$, and $\left.\kappa\right)$, and $\gamma_{1}+\gamma_{2}<1$.

In my analysis, I have assumed each production unit employs a single variable factor (labor) as well as capital. Capital is chosen before engaging in search and then remains fixed, hence playing the role of the fixed factor constraining output at the time that employment and production decisions are made. In Houthakker's language, I am working with a single variable production input and explicitly modeling the fixed factor that constrains capacity. This formulation delivers an aggregate production function with constant returns to scale, whereas the setup used by Houthakker generates a function of the variable inputs only, and it exhibits diminishing returns. We also have some differences in the way we model the heterogeneity across production units: Houthakker's production units differ in terms of their static capacity constraints (interpreted as the quantities of the unmodeled fixed production factor), whereas mine differ due to random productivities that evolve over time. But despite all these differences, note that the factor allocation rule in the search equilibrium, (8), is reminiscent of (26) and that (18) and (19) are the analogues of (27) and (28).

Another technical difference between both analyses is that by virtue of working within a 
partial equilibrium framework, Houthakker had the freedom to assume the particular shape of the heterogeneity across production units to be used in the integrals on the right sides of $(27)$ and (28). Loosely speaking, his production units could not "move around the distribution." In contrast, because I am working within the context of a dynamic equilibrium model, I do not have the freedom to choose the distribution that shows up in the aggregates. Specifically, I cannot choose $H$, which corresponds to Houthakker's $\varphi$, because $H$ is not a primitive. I can only specify $G$. This introduces an additional layer of complexity, since the mapping between the primitive distribution of shocks, $G$, and the equilibrium distribution, $H$, depends on equilibrium forces. ${ }^{23}$

From the perspective of understanding the determinants of TFP, a crucial difference is that the shift parameter in Houthakker's production function (what he called $C$ ) is solely a function of the parameters in the primitive productivity distribution. But in the model I developed above, government policy can — through the agents' decisions - affect the multiplicative factor in front of the aggregate production function.

At this point it may be useful to stress that in this context, by an "aggregate production function" I mean an equilibrium relationship between measured aggregate inputs and output. In terms of (22), the fact that this is an equilibrium relationship implies that in general, a change in policy parameters (e.g., unemployment benefits) will not affect $\gamma$ but will affect $N, K_{e}$, and $A$. Intuitively, one may regard $A$ as an additional "factor," which has to do with how production is organized across micro units, and naturally this factor responds to policy, much as, say, hours worked would. The contemporary reader may wonder: If this is what an aggregate production function is, then what does "aggregation" mean?

The classic problem of aggregation of production functions consists of finding an aggregate relationship between an output aggregate and input aggregates. For example, suppose the

\footnotetext{
${ }^{23}$ In the model of Section 2, with independently and identically distributed productivity shocks, $H$ is simply the truncation of $G$. Since the Pareto family is closed under truncations, the mapping between the primitive $G$ and the equilibrium distribution $H$ is simple, as is evident from (20) and (21). This simplicity is retained in the formulation with serially correlated shocks that I work out in Section 8.1. The mapping between $G$ and $H$ is less straightforward in other cases; for example, see the results in Section 8.2.
} 
production function for the $j^{\text {th }}$ productive unit is $y_{j}=f^{j}\left(K_{j}, N_{j}\right)$. Assume capital and labor are homogeneous, and let $K=\sum_{j} K_{j}$ and $L=\sum_{j} N_{j}$. The problem of aggregation consists of finding conditions under which there exists a function $F$ such that $\sum_{j} y_{j}=F(K, N)$. It has been known since the late 1940's that if nothing else is said, the general conditions under which such aggregation can be performed are extremely restrictive. Except in some very special cases, in an economy with multiple production units, the amount of total output that can be obtained from different levels of total inputs is not unique; the distribution of the inputs matters. ${ }^{24}$ For this reason, the aggregation literature has traditionally defined an aggregate production function as a relationship between the amount of total output produced, not from any arbitrary levels of total inputs, but for levels of total inputs that satisfy some conditions. For example, the aggregate production function in Houthakker (1955-1956) is a relationship between aggregate output and aggregate inputs - given that the allocation of inputs across production units is consistent with profit maximization. Similarly, the aggregate production function (22) is a relationship between aggregate output and aggregate inputs - given that the allocation of inputs across production units is consistent with equilibrium. ${ }^{25}$

\subsection{Measurement}

The aggregate production function in (22) expresses output as a function of the aggregate number of hours worked, $N$, and the total amount of capital hired by firms with filled jobs, $K_{e}$. The aggregate is Cobb-Douglas despite fixed proportions in the micro-level technologies. This result obtains when there is hoarding and capital utilization is imperfectly measured, i.e., when the capital stock included as an argument in the aggregate production function is the one in place in firms that are employing workers, although some of it may be idle. Notice that if there is no hoarding in equilibrium (i.e., if $\mu=R$ ), then $N=K_{e}$ and $F\left(K_{e}, N\right)=A K_{e}^{\gamma} N^{1-\gamma}=A K_{e}$. If there is hoarding but utilization is perfectly measured, then aggregate output is again linear

\footnotetext{
${ }^{24}$ To see this point, try to add up two identical Cobb-Douglas production functions without further restrictions. For more on this, see Fisher (1969) and references therein.

${ }^{25}$ These notions should be familiar: The standard aggregation result for the one-sector growth model with constant-returns technologies also relies on a distribution of inputs consistent with firm profit maximization.
} 
in the relevant capital stock. To see this explicitly, let $K_{p}$ denote the capital stock being used in production, that is, $K_{p}=[1-H(\mu)] K_{e}$. Then it follows from (18) that $Y=A^{\prime \prime} K_{p}$, with $A^{\prime \prime} \equiv E_{H}(x \mid x \geq \phi)$. So the imperfect measurement of capital utilization as described, coupled with some degree of hoarding, causes the aggregate to look Cobb-Douglas in capital and hours despite fixed proportions in the micro production functions. Since having firm-worker pairs that sometimes choose to be inactive affects the shape of the aggregates, in Appendix B I derive conditions on primitives such that the equilibrium will exhibit this labor-hoarding property.

Although hoarding and the measurement of factor utilization have implications for the shape of the aggregate production function, one should not conclude that the theory predicts only productivity differentials due to hoarding and mismeasured utilization. For example, even if there is no hoarding at all, the TFP term is equal to $\alpha R /(\alpha-1)$, the mean of the productivity distribution of active firms. Of course, the way inputs are measured in general, and in particular the extent to which their utilization rates are correctly measured, will affect the TFP factor when it is measured as a residual. I turn to this issue next.

Consider how the observed level of TFP is affected by the different ways of measuring aggregate inputs that can be found in the growth accounting literature. The measure of capital input used by Hall and Jones (1999), for example, did not adjust for utilization. This means that something like $K$ instead of $K_{e}$ was used as an input in the aggregate production function, implying $\hat{F}(K, N)=\hat{A} K^{\gamma} N^{1-\gamma}$, with $\hat{A}=\left[\frac{1-u}{1-(1-\theta) u}\right]^{\gamma} A$. In addition, Hall and Jones (1999) report they did not have data on hours per worker for all countries in their sample, so they used the number of employed workers instead of hours worked as a measure of labor input. Letting $E=1-u$ denote employment and using (17), the number of hours worked is $N=(R / \mu)^{1 / \gamma} \frac{K E}{1-(1-\theta) u}$, so- from the standpoint of the theory developed here - the aggregate relationship between inputs, output, and TFP that they measured was $\tilde{F}(K, E)=\tilde{A} K^{\gamma} E^{1-\gamma}$, with $\tilde{A}=\left[\frac{(R / \mu)^{1 / \gamma} K}{1-(1-\theta) u}\right]^{1-\gamma} \hat{A}$. In Section 7 I will quantify the impact that each of the labor market policies introduced in Section 5 has on the TFP residual for these different ways of measuring factor inputs. 


\subsection{Standard Growth Accounting}

Macroeconomists interested in identifying the sources of differences in output per worker find it useful to relate aggregate measures of output to aggregate measures of capital and labor through an aggregate production function. As a matter of accounting, this abstraction allows one to attribute the observed variation in output levels to differences in the quantities of the inputs and to differences in an unexplained TFP residual. The overwhelming majority of these growth accounting exercises adopts a Cobb-Douglas specification for the aggregate production function. ${ }^{26}$

Virtually all these growth accounting exercises rest either explicitly or implicitly on the premise that the model with frictionless markets and competitive pricing is a good approximation to the actual economy. But what if it is not? What if the actual economy lay closer to a model where labor markets are frictional and prices determined by bargaining? Would adopting this alternative view - as many economists have over the last 20 years to study various macro questions - imply that the standard growth accounting exercises that adopt a Cobb-Douglas specification, e.g., Hall and Jones (1999), Klenow and Rodríguez-Clare (1997), Parente and Prescott (2000), and many others, are misspecified in ways that render them completely uninformative?

On a positive note, the analysis of Section 4 offers a relatively standard search model — with its underlying meeting frictions, simple fixed-proportions micro-level production technologies, and prices determined by bargaining - according to which the standard growth accounting exercises that adopt a Cobb-Douglas specification for the aggregate technology have in fact chosen the right functional form. The problem is, of course, that the direct mapping between factor income shares and the elasticities in the aggregate production function that exists in the

\footnotetext{
${ }^{26}$ The most common justification for adopting this particular specification is that, at least for some countries, notably the United States and the United Kingdom, factor income shares have remained roughly constant over long periods of time, despite rather large secular changes in factor prices. (See Figure 1 in Gollin, 2002, and the bottom panel of Figure 1 in Blanchard, 1997.) And, as pointed out by the eponym Cobb and Douglas (1928), the specification implies constant factor shares of income if factors are paid their marginal products.
} 
frictionless model with competitive prices that underlies the standard growth accounting exercises is distorted in the search economy. Suppose, for example, that a growth accountant assumes $Y=B K_{e}^{\sigma} N^{1-\sigma}$ and works under the presumption that factor markets are competitive and frictionless, when instead the actual economy is as described in Sections 2-4, so that $Y=A K_{e}^{\gamma} N^{1-\gamma}$ is in fact the correct relationship between aggregate output and inputs. The TFP residual measured by this accountant would be $B=[1-H(\mu)]^{\sigma-\gamma} A .^{27}$ Since he uses the competitive model to think about the actual economy, the accountant will be inclined to set $\sigma$ equal to the capital share in income from his data. In contrast, an accountant who organizes his thoughts using the model in Section 4 will be led to look at purely technological features of the actual economy to pin down $\gamma \cdot{ }^{28}$ The relationship between the size and direction of the bias and these fundamentals is simple: The absolute size of the bias increases in the degree of hoarding. No hoarding implies the accountant with the "wrong" (competitive) model would still recover the "right" TFP factor. The direction of the bias, when there is one, depends on the sign of $\sigma-\gamma$. If there is some hoarding, and the capital share in the data is smaller than the technological parameter $\gamma$, then the accountant guided by the competitive model will overestimate the size of the true productivity residual.

In summary, the theory developed here provides foundations - which rest on a model with a frictional labor market and noncompetitive wages - for the Cobb-Douglas specification of the aggregate production function commonly used by macroeconomists for growth accounting. The theory implies a mapping between observables and the elasticity parameter in the aggregate that is different from that implied by the competitive model, and it can be used to assess the implications of this difference for the size of the measured residual.

The emphasis throughout the paper is on understanding the determinants of the TFP

\footnotetext{
${ }^{27}$ Here I have assumed that the accountant measures only the capital in place in firms that have positive employment, $K_{e}$, but it may be more realistic to suppose he instead measures the total capital stock, $K$. If this is the case, the measured residual would be $B=\left[\frac{1-u}{1-(1-\theta) u}\right]^{\sigma}[1-H(\mu)]^{\sigma-\gamma} A$.

${ }^{28}$ Recall that $\gamma=1 / \alpha$ is a parameter of the underlying distribution of shocks. So, for example, the growth accountant may attempt to infer it from the distribution of value added across production units. I thank Esteban Rossi-Hansberg for suggesting this possibility.
} 
residual, and factor income shares are not central to this issue from the standpoint of the theory. However, factor income shares play a crucial role in standard growth accounting, so the reader may feel that some remarks on the nature of the factor shares in the model may be in order. In this model, factor income shares are not immutable technological constants: they depend not only on technological considerations but also on labor market policies or anything else that affects the division of rents between workers and employers. For example, policies that increase the unemployment rate will tend to decrease the labor share. ${ }^{29}$

I conclude this section by commenting on where this paper stands relative to the literature that uses growth accounting to frame some fundamental questions in development economics. In the model developed here, differences in TFP arise from composition effects, i.e., through the way production inputs, particularly hours of work, are allocated across micro production units. This feature of the theory naturally leads one to think of institutions that may distort this allocation of resources, and this is where labor-market policies come in as a natural suspect. Note that the mechanisms at work in this paper allow two economies to exhibit different levels of TFP even if production units in both have access to the same technology and are subject to identical shocks. But the world's richest and poorest countries seem to have very different production technologies in place, so trying to explain 30-fold output-per-worker differences keeping technologies the same and relying exclusively on policies that only distort the allocation

\footnotetext{
${ }^{29}$ Blanchard (1997) documents the decline in the labor share for continental European countries (France, Germany, Italy, and Spain) during 1970-1995. He offers changes in policies and institutional considerations that affected the relative bargaining positions of employers and workers as plausible explanations. Interestinglyfrom the point of view of the competitive model-nonconstant factor shares imply that the aggregate production function could not be Cobb-Douglas. But from the point of view of the aggregative model developed here, this conclusion does not follow: it can exhibit nonconstant factor shares and, with a Pareto distribution of idiosyncratic productivity shocks, it can still aggregate up to a Cobb-Douglas production function. To illustrate, let $s_{w}$ and $s_{k}$ denote the labor and capital shares, respectively, and consider a version of the basic model in which $\phi$ indexes a worker's disutility from work (see Appendix B), $b=\tau_{b} k$, and there is no fixed cost (i.e., $C=0$ ). Then,

$$
s_{w}=\beta+\xi(1-\beta)-z_{k} \frac{K_{e}}{Y}+z_{n} \frac{N}{Y} \quad \text { and } \quad s_{k}=1-s_{w},
$$

where $z_{k} \equiv[1-(1-\xi)(1-\beta)] c-(1-\xi)\left[\beta c \theta+(1-\beta) \tau_{b}\right], z_{n} \equiv(1-\xi)(1-\beta) \phi$, and $\xi$ is an accounting parameter that denotes the fraction of the firm's rents imputed as labor income. So, for example, if $\xi=1$, then $s_{k}=\frac{c K_{e}}{Y}=\frac{c}{F_{1}\left(K_{e}, N\right)} \gamma$.
} 
of resources (labor or otherwise), would seem misguided. ${ }^{30}$ In the following section I calibrate the model economy and show that - if assessed within the context of a homogeneous set of countries, e.g., the Organisation for Economic Co-operation and Development (OECD) - the magnitude of the effects of labor market policies on the level of measured TFP can in some cases be significant.

\section{Quantitative Analysis}

Consider an extension of the model of Section 5 where upon meeting, the firm-worker match draws the initial productivity from a cumulative distribution function with $G_{0}(x)=1-\left(\frac{\varepsilon_{0}}{x}\right)^{\alpha}$ if $\varepsilon_{0} \leq x$ and $G_{0}(x)=1-\left(\frac{\varepsilon_{0}}{x}\right)^{\alpha}=0$ otherwise, where $\varepsilon_{0}>0$. Subsequent draws are from (20). As I show in Appendix B (Lemma 3), for this case the equilibrium cumulative distribution function of active firms is given by (21). The job-creation and job-destruction conditions are given by (24) and (25), but with $\varepsilon_{0}$ replacing $\varepsilon$ in $(25) .{ }^{31}$

I will follow the bulk of the search and matching labor literature in assuming that the meeting rate of a vacancy is $q(\theta)=\theta^{-\eta}$, with $\eta \in(0,1)$. To calibrate this model to the United States, I set $\tau_{f}=\tau_{h}=\tau_{e}=0$, which is meant to reflect its relatively flexible labor market regulations. Since the model will generate endogenous separations, I also set $\delta=0$. This leaves

\footnotetext{
${ }^{30}$ In order to make the quantitative leap to development accounting within the context of this model, one may have to formalize the notion that the policies considered not only distort the allocation of inputs but also hinder the adoption of better technologies. Although they focus on monopoly rights in their formal modeling of barriers to technology adoption, Parente and Prescott (2000) mention several labor-market policies as examples of "barriers to riches": "In India, for example, firms with more than 100 workers must obtain the government's permission to terminate any worker, and firms of all sizes are subject to state certification of changes in the tasks associated with a job" (pp. 107-108). "Another way the state protects the monopoly rights is by requiring large severance payments to laid-off workers." "Also in India, regulations require certain firms to award workers with lifetime employment and require firms with more than twenty-five workers to use official labor exchanges to fill any vacancy" (p. 108). "In Bangladesh, for example, private buyers of the state-owned jute mills were prohibited for one year from laying off any of the workforce they inherited. After one year, a worker could be laid off but not without a large severance payment." Parente and Prescott use these examples as instances of policies that can lower TFP by making technology adoption costly, whereas I emphasize that these policies have a direct impact on the level of TFP through composition effects - even for given technology. See footnote 1 for some issues that the model developed here may be well suited for.

${ }^{31}$ Varying $\varepsilon_{0}$ relative to $\varepsilon$ will help calibrate the model by allowing me to vary the job-creation condition in much the same way as the usual cost of posting vacancies does in the standard formulation of the MortensenPissarides model. Recall that in my formulation, a firm's only flow cost from searching is having to pay rental $c$ on the capital $k$, and $c$ will be pinned down by the interest rate.
} 
10 free parameters: $r, c, \varepsilon, \eta, \beta, \alpha, \lambda, \varepsilon_{0}, \tau_{b}$, and $\phi$. I normalize the time period to be a quarter and set $r=c=0.012$, which corresponds to an annual discount factor of 0.953. I also normalize $\varepsilon=1$. I set $\eta=0.72$, which is close to the upper bound of the range reported by Petrongolo and Pissarides (2001), and let $\beta=\eta$ so that there would be no inefficiencies in the absence of policy. $^{32}$

Several studies point out that various measures of firm size are well approximated by a Pareto distribution with a curvature parameter near 1. Axtell (2001), for example, documents this fact using the number of employees and sales as measures of firm size. The model I am calibrating is in some ways too stark: for example, each firm employs a single worker, and it does not distinguish between output and sales. But notice that the equilibrium distribution of output across active firms is Pareto with curvature parameter $\alpha$. These considerations may suggest a natural strategy to calibrate $\alpha$. However, since the purpose here is mainly to explore the properties of the model for reasonable parametrizations rather than to devise a definitive calibration strategy, I will report results for three values of $\alpha: 1.1,3 / 2$, and 3 . The remaining parameters $\left(\lambda, \varepsilon_{0}, \tau_{b}, \phi\right)$ are calibrated so that in the stationary equilibrium, the model implies that (i) employment spells last 2.5 years on average; (ii) an unemployed worker finds a job with probability 0.45 per month; (iii) the replacement rate is $11 \%$; and (iv) the fraction of hoarded employed workers is relatively small, in particular $0.05 .^{33}$

For the benchmark case $(\alpha=3 / 2)$, the values of the calibrated parameters are $\lambda=1.23$, $\varepsilon_{0}=0.6, \tau_{b}=0.22$, and $\phi=1.095$. The implied means of the distributions $G$ and $G_{0}$ are 3 and 1.84 , respectively. In the equilibrium, $R=1.058$, and the average wage is $\bar{w}=2.014$.

\footnotetext{
${ }^{32}$ Here I am following Shimer (2005), who estimates $\eta=0.72$ and also sets $\beta=\eta$. See Hagedorn and Manovskii (2005) for an alternative calibration strategy. In Appendix B, I report results for my own alternative calibration strategy, which instead sets $\beta$ to match the labor share.

${ }^{33}$ For observations (i) and (ii), see Shimer (2005). Observation (iii) corresponds to the average value over the first five years of unemployment expressed as a percentage of previous tax earnings for a single worker reported by the OECD Jobs Study (OECD, 1994). Most of the empirical work on labor hoarding has focused on its cyclical properties and their implications for the Solow residual-based measures of technology shocks, and for the extent to which technology shocks account for the volatility of aggregate output (e.g., Burnside, Eichenbaum, and Rebelo, 1993). I have not been able to find empirical estimates of the average level of labor hoarding in my perusal of the literature. Consequently, target (iv) is not based on any hard data, but just meant to be illustrative.
} 


\begin{tabular}{|c|c|c|c|c|c|c|c|c|}
\hline & & $\overline{\overline{\tau_{i} / \bar{w}}}$ & $\bar{D}$ & $\bar{u}$ & $\overline{A_{\tau_{i}} / A_{0}}$ & $\overline{\hat{A}_{\tau_{i}} / \hat{A}_{0}}$ & $\tilde{A}_{\tau_{i}} / \tilde{A}_{0}$ & $\bar{~} \overline{H(\phi)}$ \\
\hline \multicolumn{2}{|c|}{ baseline } & 0.11 & 10 & 0.069 & 1.000 & 1.000 & 1.000 & 0.050 \\
\hline \multirow[t]{2}{*}{$\tau_{b}$} & 0.25 & 0.12 & 7 & 0.096 & 1.023 & 0.847 & 0.787 & 0.017 \\
\hline & 0.19 & 0.10 & 18 & 0.038 & 0.975 & 1.318 & 1.497 & 0.086 \\
\hline \multirow{2}{*}{$\overline{\tau_{f}}$} & 0.02 & 0.01 & 16 & 0.041 & 0.977 & 1.278 & 1.432 & 0.082 \\
\hline & 0.04 & 0.02 & 54 & 0.012 & 0.954 & 1.973 & 2.717 & 0.114 \\
\hline \multirow[t]{2}{*}{$\tau_{e}$} & 0.02 & 0.01 & 14 & 0.048 & 0.983 & 1.183 & 1.277 & 0.074 \\
\hline & 0.04 & 0.02 & 23 & 0.030 & 0.968 & 1.463 & 1.745 & 0.095 \\
\hline \multirow[t]{2}{*}{$\overline{\tau_{h}}$} & 0.02 & 0.01 & 7 & 0.096 & 1.023 & 0.840 & 0.777 & 0.017 \\
\hline & 0.03 & 0.02 & 6 & 0.108 & 1.034 & 0.786 & 0.706 & 0.001 \\
\hline
\end{tabular}

Table 1: Baseline results

Table 1 reports the effects of small changes in the policy parameters $\tau_{b}, \tau_{f}, \tau_{e}$, and $\tau_{h}$, on the average duration of a job, $D=[\lambda G(R)]^{-1}$, the unemployment rate, $u$, the three measures of TFP described in Section 6.2 (i.e., $A=\frac{Y}{K_{e}^{\gamma} N^{1-\gamma}}, \hat{A}=\frac{Y}{K^{\gamma} N^{1-\gamma}}$, and $\tilde{A}=\frac{Y}{K^{\gamma} E^{1-\gamma}}$ ), and the degree of labor hoarding. I use subscripts to index the various policy scenarios, e.g., $A_{0}$ denotes the level of TFP at the baseline with no policy, and $A_{\tau_{i}}$ denotes the level of TFP under policy $\tau_{i}$, for $i=b, f, e, h$. The first row just shows that the model hits the calibration targets: the replacement rate $\tau_{b} / \bar{w}$ is exactly $11 \%$, and on average a job lasts 10 quarters, which, together with a probability of 0.45 per month that an unemployed worker finds a job, implies an unemployment rate of $6.9 \%$. Also, $H(\phi)=.05$, indicating that in the equilibrium $5 \%$ of the firms hoard labor.

Increasing the replacement rate from $11 \%$ to $12 \%$ (which is achieved by increasing $\tau_{b}$ from 0.22 to 0.25$)$ shifts the job-destruction condition upward as in the top-right panel of Figure 1. The increase in $R$ causes the expected duration of a job to fall to seven quarters, and this, together with the reduction in job creation induced by the fall in $\theta$, causes the unemployment rate to increase to $9.6 \%$. According to the first measurement convention, $A$, for which the capital stock in the aggregate production function is $K_{e}$ and hours worked are measured accurately, the $1 \%$ increase in the replacement rate causes a $2.3 \%$ increase in $R$ and hence a $2.3 \%$ increase in TFP. 
The measurement issues discussed in Section 6.2, however, are critical. For example, under the second measurement convention, $\hat{A}$, for which the capital stock in the aggregate production function is $K$ and hours worked are measured accurately, the $1 \%$ increase in replacement rate causes an $18 \%$ reduction in the measured residual. This method is picking up such a large reduction in TFP because after the increase in unemployment benefits, the unemployment rate is significantly higher, and hence a significantly higher fraction of the capital stock that is counted as an aggregate input is now sitting idle in unmatched firms. Recall that the third measurement convention, $\tilde{A}$, is the one that uses $K$ and employment (instead of hours worked) as inputs. According to this measure, TFP falls by $27 \%{ }^{34}$

Intuitively, the second way to measure inputs perfectly measures the utilization rate of labor, but does not measure the utilization rate of capital at all (it cannot even distinguish machines sitting in empty job slots from those matched to workers). And the third way does not adjust for the utilization rate of either factor. In terms of labor, it counts both workers who are employed and supplying hours as well as those who are employed but not supplying hours. The policy causes unemployment to rise, which reduces the economy-wide utilization rate of capital and labor. Thus, the measures that don't adjust for utilization capture a sharp reduction in the TFP residual.

Introducing a firing tax that corresponds to $1 \%$ of the average quarterly wage reduces unemployment by 2.8 percentage points. TFP as measured by $A$ drops by $2.3 \%$ but rises when measured by $\hat{A}$ or $\tilde{A}$, by $28 \%$ and $43 \%$, respectively. ${ }^{35}$ The intuition for the first measure is clear: the productivity of the average active firm is lower because the policy makes separations more costly, and hence lower-quality matches survive in the presence of firing restrictions. The large increases in aggregate productivity as measured by $\hat{A}$ and $\tilde{A}$ are again due to the fact that

\footnotetext{
${ }^{34}$ The decline is larger for the third measure because the total number of hours worked, averaged over all employed workers, falls in response to the increase in $\tau_{b}$.

${ }^{35}$ In a recent paper, Chari, Restuccia, and Urrutia (2005) develop a matching model with dynamic labor contracts in which firms provide general training to workers who cannot commit to staying with the firm. Within this setup, they find that firing costs can increase measured productivity, and they argue that this result is borne out by European data.
} 
the unemployment rate is lower, and hence the utilization rate of the factors is higher with the policy. The magnitudes of the effects of the remaining policies are similar. ${ }^{36}$

Tables 2 and 3 in Appendix B report results for other two parametrizations: $\alpha=1.1$ and $\alpha=3$. Essentially, the policy effects are much larger for the former and much smaller for the latter. Table 4 in Appendix B reports results for another calibration strategy. There, instead of following Shimer (2005) in setting $\beta=\eta=0.72$, I keep $\eta=0.72$ and calibrate $\beta$ jointly with $\left(\lambda, \varepsilon_{0}, \tau_{b}, \phi\right)$ so that the model also reproduces a labor share of 0.66 . The general message from these exercises is that (i) the value of the curvature parameter of the Pareto distribution, $\alpha$, is a critical determinant of the size of the effects that policy can have on TFP through composition effects (the effects tend to get larger as $\alpha$ approaches 1), and (ii) if factors are measured relatively inaccurately (e.g., when the TFP residual equals $\hat{A}$ or $\tilde{A}$ ), the policy effects on TFP are much larger and have the opposite sign than if the inputs are measured accurately (e.g., when the TFP residual equals $A$ ).

\section{Extensions}

In this section I extend the basic model to the case of serially correlated shocks and derive two additional aggregation results.

\subsection{Correlated Shocks}

Section 4 established that, with some mismeasurement and equilibrium hoarding, the standard search model of the labor market with a particular structure of shocks generates a relationship between aggregate inputs and output that looks exactly like the standard Cobb-Douglas relation typically used in growth accounting exercises. The fixed cost $C(x, \phi)$ was introduced in Section 2 as a simple device to avoid "flat spots" in the value functions, and this made it possible for

\footnotetext{
${ }^{36}$ But note that financing the employment subsidy is relatively more costly than financing the hiring subsidy, since the former is paid out continuously to existing matches, whereas the latter just entails a one-time payment when the match is first formed.
} 
the equilibrium to exhibit hoarding. ${ }^{37}$ Here I show that by extending the model in a natural way, one can drop the fixed cost without affecting the main results. To this end, I generalize the productivity process by allowing for serially correlated shocks: when a match of productivity $x$ suffers a change, the new value $x^{\prime}$ is a draw from the fixed distribution $G\left(x^{\prime} \mid x\right)$. Assuming $G\left(x \mid x_{1}\right)<G\left(x \mid x_{0}\right)$ if $x_{0}<x_{1}$ allows idiosyncratic shocks to be positively correlated through time. For this case, the cross section of productivities evolves according to

$$
\begin{aligned}
\frac{d}{d t}\left[\left(1-u_{t}\right) H_{t}(x)\right]= & \lambda\left(1-u_{t}\right) \int_{x}^{\infty}\left[G(x \mid s)-G\left(R_{t} \mid s\right)\right] d H_{t}(s) \\
& +\theta q(\theta) u_{t} \int_{-\infty}^{\infty}\left[G(x \mid s)-G\left(R_{t} \mid s\right)\right] d H_{t}(s) \\
& -\lambda\left(1-u_{t}\right) \int_{-\infty}^{x} G\left(R_{t} \mid s\right) d H_{t}(s) \\
& -\lambda\left(1-u_{t}\right) \int_{-\infty}^{x}[1-G(x \mid s)] d H_{t}(s)-\delta\left(1-u_{t}\right) H_{t}(x) .
\end{aligned}
$$

The first term accounts for the matches with productivities above $x$ that get innovations below $x$ but above $R_{t}$. The newly formed matches that start off with productivities no larger than $x$ are in the second term. Notice the assumption that upon contact, the worker and firm draw their productivity level from the density corresponding to the average productivity among active matches. ${ }^{38}$ The third term is the number of matches in the interval $\left[R_{t}, x\right]$ that get shocks below $R_{t}$ and are destroyed. The fourth term accounts for the number of matches in the same interval that "move up" by virtue of having drawn productivities larger than $x$. The last term

\footnotetext{
${ }^{37}$ For $C(x, \phi)=0, \pi(x)=[\max (x-\phi, 0)-c] k-w(x)$, so $\pi(x)$ is flat up to $\phi$ and then rises with slope $k$. It is easy to show that in this case, $J(x)$ is also flat up to $\phi$ and then rises with slope $\frac{k}{r+\delta+\lambda}$. Note that since $R$ is defined by $J(R)=0$, this implies that generically the equilibrium with endogenous destruction will have $\phi<R$; i.e., there is no hoarding except for the knife-edge case in which $R$ is indeterminate. Ruling out these types of flat spots in $J$ allows for the possibility that $R<\phi$ in an equilibrium with endogenous destruction. See Appendix B for details.

${ }^{38}$ When shocks are independently and identically distributed, one can specify that new matches draw $z$ from $G(z)$ just as active matches do when forced to update their shock. However, with correlated shocks active matches with state $z$ draw the new shock $z^{\prime}$ from $G\left(z^{\prime} \mid z\right)$. Since vacancies and unemployed workers have no productivity attached to them, I assume their initial draw $z^{\prime}$ is from the average density $\int G\left(z^{\prime} \mid z\right) d H(z)$. As a way of motivating this, imagine - as do Mortensen and Pissarides (1994) - that firms must irreversibly adopt a "technology" to engage in production. The present specification then means that they draw their technology at random from all those active at the time the match is created. Jeffrey Campbell pointed out to me that Conlisk (1989) uses a similar assumption to determine the productivity of newly created plants in a model of technical change.
} 
accounts for matches in the interval $\left[R_{t}, x\right]$ that are destroyed for exogenous reasons. Imposing steady states and rearranging yields

$$
H(x)=\left[\frac{\lambda}{\delta+\lambda}+\frac{\theta q(\theta) u}{(\delta+\lambda)(1-u)}\right] \int[G(x \mid s)-G(R \mid s)] d H(s) .
$$

The steady-state unemployment rate is given by

$$
u=\frac{\delta+\lambda \int G(R \mid s) d H(s)}{\delta+\lambda \int G(R \mid s) d H(s)+\theta q(\theta) \int[1-G(R \mid s)] d H(s)} .
$$

Using this expression, the steady-state cross-sectional productivity distribution becomes

$$
H(x)=\frac{\int[G(x \mid s)-G(R \mid s)] d H(s)}{\int[1-G(R \mid s)] d H(s)},
$$

a natural generalization of (3).

The problem of a searching firm is now summarized by

$$
r V=-c k+q(\theta) \iint \max [J(z)-V, 0] d G(z \mid x) d H(x) .
$$

Again, entry of firms will occur until all rents are exhausted, so $r V=0$ in equilibrium. The value of a filled job with productivity $x$ is

$$
r J(x)=\pi(x)+\lambda \int \max [J(z)-V, 0] d G(z \mid x)-(\delta+\lambda)[J(x)-V],
$$

where $\pi(x)=x \min (n, k)-\phi n-c k-w(x)$. Flow profit $\pi(x)$ is the residual remaining after the wage $w(x)$ and all other costs of production have been paid out. This formulation has only two such costs: the rental rate, $c k$, and the variable cost, $\phi n .^{39}$ The choice of hours that solves the bargaining problem is still given by (8), and hence $\pi(x)=y(x)-w(x)$, where $y(x) \equiv[\max (x-\phi, 0)-c] k$ is output net of the variable cost and the rental on capital.

The values of unemployment and employment to a worker are

$$
\begin{aligned}
r U & =b+\theta q(\theta) \iint \max [W(z)-U, 0] d G(z \mid x) d H(x) \\
r W(x) & =w(x)+\lambda \int \max [W(z)-U, 0] d G(z \mid x)-(\delta+\lambda)[W(x)-U],
\end{aligned}
$$

\footnotetext{
${ }^{39}$ Here I model $\phi n$ as a cost borne by the firm, but Appendix B shows that this formulation is equivalent to one in which $\phi n$ is instead the disutility the worker experiences from working $n$ hours.
} 
where $w(x)$ is still characterized by (9). Letting $S(x)=J(x)+W(x)-U$ denote the surplus from a match, notice that (9) implies $J(x)=(1-\beta) S(x), W(x)-U=\beta S(x), \pi(x)=$ $(1-\beta)[y(x)-r U]$ and

$$
w(x)=\beta y(x)+(1-\beta) r U
$$

Combining (9) with (32), (33), and (34) yields

$$
(r+\delta+\lambda) S(x)=y(x)-r U+\lambda \int \max [S(z), 0] d G(z \mid x),
$$

where $r U$ is given by (10). The fact that $S^{\prime}(x)>0$ implies that there exists a unique $R$ such that $S(x)>0$ iff $x>R$. Hence matches separate (or don't form) for productivity draws below $R$. Moreover, notice that $S(x)$ is strictly increasing for all $x$ (even for $x<\phi$ ) despite the fact that $y(x)$ is flat for $x<\phi$. This is because $d G(\cdot \mid x)$ is stochastically increasing in $x$. Consequently, the equilibrium may have $R>\phi$ (no hoarding) for some parametrizations and $R<\phi$ (hoarding) for others. ${ }^{40}$

Next, I turn to the issue of aggregation. Suppose idiosyncratic shocks are draws from

$$
G(x \mid s)= \begin{cases}0 & \text { if } x<\varepsilon(s) \\ 1-\left[\frac{\varepsilon(s)}{x}\right]^{\alpha} & \text { if } \varepsilon(s) \leq x\end{cases}
$$

where $\varepsilon(\cdot)$ is a continuously differentiable function and $\alpha>1$. I introduce positively correlated shocks by assuming that $\varepsilon^{\prime}>0$ (if $\varepsilon^{\prime}=0$ the distribution reduces to (20), the special case of uncorrelated shocks). In addition, suppose there is an $\underline{\varepsilon}>0$ such that $\varepsilon(\underline{\varepsilon})=\underline{\varepsilon}$ and $\varepsilon(s)=0$ if $s<\underline{\varepsilon}$, and that $\lim _{s \rightarrow \infty} \varepsilon(s)=1+\underline{\varepsilon} \equiv \bar{\varepsilon}^{41}$

Then for $R \geq \varepsilon(s), 1-G(R \mid s)=\left[\frac{\varepsilon(s)}{R}\right]^{\alpha}$; hence, for any $x \geq R, G(x \mid s)-G(R \mid s)=$ $[\varepsilon(s) / R]^{\alpha}\left[1-(R / x)^{\alpha}\right]$. After substituting these expressions in (30), it becomes clear that the steady-state productivity distribution of active matches is still given by (21). So for this case,

\footnotetext{
${ }^{40}$ In trying to determine which parametrizations may exhibit hoarding, the logic of Figure 3 in Appendix B also applies to this case.

${ }^{41}$ An example of a function $\varepsilon(\cdot)$ satisfying all these conditions is $\varepsilon(s)=1+\underline{\varepsilon}-e^{\underline{\varepsilon}-s}$ for any $\underline{\varepsilon}>0$.
} 
the job-creation and job-destruction conditions are

$$
\begin{aligned}
\frac{\mu^{1-\alpha} R^{\alpha}}{\alpha-1}-\frac{\alpha[y(R)-r U]}{k}\left[\frac{R}{\varepsilon(R)}\right]^{\alpha} \int_{R} \frac{\varepsilon(x)^{\alpha}-\varepsilon(R)^{\alpha}}{x^{1+\alpha}} d x-\frac{(r+\delta+\lambda) c}{(1-\beta) q(\theta)}\left[\alpha \int_{R} \frac{\varepsilon(x)^{\alpha}}{x^{1+\alpha}} d x\right]^{-1}=0 \\
{\left[1-\frac{\lambda \alpha}{r+\delta+\lambda} \int_{R} \frac{\varepsilon(x)^{\alpha}-\varepsilon(R)^{\alpha}}{x^{1+\alpha}} d x\right] \frac{y(R)-r U}{k}+\frac{\lambda}{r+\delta+\lambda} \frac{\varepsilon(R)^{\alpha} \mu^{1-\alpha}}{\alpha-1}=0 . }
\end{aligned}
$$

An equilibrium is still a list $[R, \theta, H, U, w, u, K]$ such that $R, \theta$, and $H$ jointly solve (30) and the job-creation and job-destruction conditions, $r U$ is given by (10), $w$ is given by (35), and $u$ satisfies (29). In addition, the market for capital should clear, so $[1-(1-\theta) u] k=K$. Sufficient conditions can be found so that the job-creation condition slopes downward and the job-destruction condition slopes upward in $\theta-R$ space, implying a unique $(\theta, R)$ pair. A parameter restriction analogous to the one depicted in Figure 3 in Appendix B which guarantees that $\phi$ has a range of values such that $R<\phi$ can still be derived. ${ }^{42}$ Given the equilibrium pair $(\theta, R)$, the same procedure followed in Section 4 reveals that output aggregates to (22). Thus, the key aggregation result obtained in the context of the model of Section 2 does not hinge on some of the particular modeling choices made there. In particular, allowing for correlated shocks can generate hoarding, and there is no need for the fixed cost of Section 2.

\subsection{More on Aggregation}

In this subsection I prove two additional aggregation results. First, I provide another specification of primitives that delivers the same aggregation result obtained for the models of Sections 2 and 8.1. Again, this specification does not rely on the fixed cost of Section 2, so in a way it reinforces the message of Section 8.1. The second aggregation result shows how to reverse engineer a distribution of idiosyncratic shocks that gives rise to an aggregate constant elasticity of substitution (CES) production function.

\footnotetext{
${ }^{42}$ Showing that equilibria with $R<\phi$ are possible for some parametrizations is now rather tedious, so the basic idea is only outlined here. Let $\phi_{\bar{\varepsilon}}$ be the value of $\phi$ such that $\theta_{\bar{\varepsilon}}^{*}$ and $R\left(\phi_{\bar{\varepsilon}}\right)=\bar{\varepsilon}$ solve the job-creation and job-destruction conditions. Then if $\phi_{\bar{\varepsilon}}-\bar{\varepsilon}>0$, there will be an interval $\left(\phi_{\bar{\varepsilon}}, \widehat{\phi}\right)$ such that $R(\phi)<\phi$ iff $\phi \in\left(\phi_{\bar{\varepsilon}}, \widehat{\phi}\right)$. If, in addition, $\partial R(\phi) / \partial \phi>0$, then $\phi_{\bar{\varepsilon}}-\bar{\varepsilon}>0$ also implies $\bar{\varepsilon}<R(\phi)$ for all $\phi$. Finally, notice that $R>\bar{\varepsilon}$ also implies that every match faces a positive probability of being destroyed for endogenous reasons. To see why, suppose $R=\varpi<\bar{\varepsilon}$; then any match that reaches a state $s>\varepsilon^{-1}(\varpi)$ will never be destroyed endogenously.
} 
First consider the model of Section 2, but with $C=0$ and separation rates that are decreasing in the productivity of the match; i.e., $0<\delta(x)<\infty$ for all $x$, with $\delta^{\prime}<0$. The interpretation is that $\delta$ is a technological parameter: in any small time interval, with probability $\delta(x)$ the idiosyncratic productivity of the match jumps to zero and stays at that level forever. I return to the case of uncorrelated productivity draws for the remainder of the section. The distribution of active matches now evolves according to

$$
\begin{aligned}
\frac{d}{d t}\left[\left(1-u_{t}\right) H_{t}(x)\right]= & \lambda\left(1-u_{t}\right)\left[1-H_{t}(x)\right]\left[G(x)-G\left(R_{t}\right)\right]+\theta q(\theta) u_{t}\left[G(x)-G\left(R_{t}\right)\right] \\
& -\lambda\left(1-u_{t}\right) H_{t}(x) G\left(R_{t}\right)-\lambda\left(1-u_{t}\right) H_{t}(x)[1-G(x)] \\
& -\left(1-u_{t}\right) \int_{R} \delta(s) d H_{t}(s) .
\end{aligned}
$$

The value functions (5) and (7) are respectively replaced by

$$
\begin{aligned}
r W(x) & =w(x)+\lambda \int \max [W(z)-U, 0] d G(z)-[\delta(x)+\lambda][W(x)-U] \\
r J(x) & =\pi(x)+\lambda \int \max [J(z)-V, 0] d G(z)-[\delta(x)+\lambda][J(x)-V],
\end{aligned}
$$

while (4) and (6) remain unchanged. The bargaining outcome is still characterized by (8) and (9), and the value functions imply

$$
S(x)=\frac{[\max (x-\phi, 0)-c] k-r U+\lambda \int \max [S(z), 0] d G(z)}{r+\delta(x)+\lambda},
$$

which is clearly increasing in $x$ and has a kink at $\phi$, as is usual when departing from the simple fixed-cost formulation of Section 2. Plotting $S(x)$ reveals that, depending on parametrizations, two cases are possible: the kink could be above or below the horizontal axis. If it is above, then $R<\phi$ and the equilibrium exhibits hoarding. The job-creation and job-destruction conditions can be derived as usual, and an equilibrium can be summarized by the $(\theta, R)$ pair that solves them. The following result provides conditions under which this model aggregates to (22), just as in the models of Section 2 and 6.1.

Proposition 3 Suppose $\delta(x)=\delta x^{-\sigma}$ and the primitive density of shocks is

$$
d G(x)=\frac{\alpha \lambda(\alpha+\sigma) \varepsilon^{\alpha}}{\lambda(\alpha+\sigma)+\alpha \delta \varepsilon^{-\sigma}}\left(1+\frac{\delta}{\lambda} x^{-\sigma}\right) x^{-\alpha-1}
$$


for $x \geq \varepsilon$ and $d G(x)=0$ otherwise, where $\varepsilon, \delta, \sigma>0$, and $\alpha>1$. Then in equilibrium, the aggregates $Y, K_{e}$, and $N$ satisfy (22).

So far I have shown that in several versions of the Mortensen-Pissarides model, for certain distributions of the idiosyncratic shocks, aggregate output looks like a Cobb-Douglas function of the aggregate labor and capital inputs. What follows generalizes the previous results by characterizing the distribution of shocks that gives rise to an aggregate CES production function.

Suppose the primitive distribution of shocks, $G$, is given by

$$
G(x)= \begin{cases}0 & \text { if } x<\varepsilon \\ 1-\left[\frac{1}{\sigma}\left(\frac{x}{\varepsilon}\right)^{\frac{\rho}{1-\rho}}-\frac{1-\sigma}{\sigma}\right]^{-1 / \rho} & \text { if } \varepsilon \leq x,\end{cases}
$$

with $\varepsilon>0$ and $\rho, \sigma \in(0,1) .{ }^{43}$ Substituting (37) into (3), one sees that for any $R \geq \varepsilon$, the steady-state productivity distribution of active matches is

$$
H(x)=1-\kappa\left[\frac{1}{\sigma}\left(\frac{x}{\varepsilon}\right)^{\frac{\rho}{1-\rho}}-\frac{1-\sigma}{\sigma}\right]^{-1 / \rho}
$$

if $R \leq x$, and $H(x)=0$ if $x<R$, with $\kappa \equiv[1-G(R)]^{-1}$. Following a strategy similar to that of Levhari (1968), one can establish the following result.

Proposition 4 If the primitive distribution of the idiosyncratic shocks is given by (37), then in equilibrium, the aggregates $Y, K_{e}$, and $N$ satisfy $Y=B\left[\sigma \bar{A} K_{e}^{\rho}+(1-\sigma) N^{\rho}\right]^{1 / \rho}$, with $B=\frac{\varepsilon}{1-\sigma}$, and $\bar{A}=\left[\frac{1}{\sigma}\left(\frac{R}{\varepsilon}\right)^{\frac{\rho}{1-\rho}}-\frac{1-\sigma}{\sigma}\right]$.

In this case, all the characteristics of the labor market as summarized by $R$ affect the measured productivity of inputs asymmetrically. ${ }^{44}$ Notice that as $\rho \rightarrow 0,(37)$ approaches the Pareto distribution in (20) with parameters $\varepsilon$ and $\alpha=1 / \sigma$. So in this sense, the CES aggregate in Proposition 4 approaches the Cobb-Douglas aggregate in (22) as the elasticity of substitution $1 /(1-\rho)$ approaches unity. ${ }^{45}$

\footnotetext{
${ }^{43}$ Under these conditions, $G^{\prime}(x) \geq 0$ and $\lim _{x \rightarrow \infty} G(x)=1$, so $G$ is a proper cumulative distribution function.

${ }^{44}$ If the aggregation were performed using (37) instead of its truncation, then the aggregate would instead be $Y=\frac{R}{1-\sigma}\left[\sigma K_{e}^{\rho}+(1-\sigma) N^{\rho}\right]^{1 / \rho}$. However, no primitive density has $(37)$ as its truncation.

${ }^{45}$ Notice, however, that the truncation of (37) does not approach (21) as $\rho \rightarrow 0$. That is, even though the
} 


\section{Concluding Remarks}

This paper developed a theory of TFP differences based on the interaction between institutions and the microeconomics underlying the aggregate production function. The analysis focused on a precise class of institutions, namely, labor-market policies as measured by the magnitudes of hiring subsidies, employment subsidies, unemployment benefits, and firing taxes. In the model, firm-level technologies are subject to idiosyncratic shocks that induce a cross-sectional distribution of productivities. Through their effects on job-creation and job-destruction decisions, labor-market policies affect the productivity composition of active firms - and ultimately TFP.

By thinking of the aggregate production function as an equilibrium relationship between aggregate inputs and output arising from the aggregation of heterogeneous micro-level production units, I was able to show analytically how the measured level of TFP-usually a "black box" that drives cross-country income differences - depends on primitive technological parameters, government policies, and other fundamental features of the economic environment. This approach may prove useful in interpreting aggregate productivity data and can serve as a guide to uncovering sources of cross-country differences in measured TFP.

primitive distribution approaches a Pareto, its truncation does not limit a truncated Pareto. This is because the density in (37) is not "closed" under truncations (as, for example, the Pareto and the exponential distributions are). This "discontinuity" introduced by the truncation is the reason why if we take the limit on the truncated cumulative distribution function or on the CES aggregate directly, we don't obtain exactly (22). 


\section{A Appendix}

\section{Proof of Proposition 1.}

In $R-\theta$ space, the slopes of the job-destruction and job-creation conditions (24) and (25) are

$$
\frac{1-\beta}{\beta c}\left[1-\frac{\lambda(\varepsilon / R)^{\alpha}}{r+\delta+\lambda}\right] \text { and } \frac{(1-\beta) \theta q(\theta)(\varepsilon / R)^{\alpha} \tau(R)}{-c \eta(\theta)(r+\delta+\lambda) R}
$$

respectively, where $\tau(R)=R+\alpha(r+\delta+\lambda)\left(\tau_{h}-\tau_{f}\right)$. Note that for all $R \geq \varepsilon$, the destruction condition slopes upward and the creation condition slopes downward. If $\phi=\phi_{\epsilon}$, then (24) and (25) have a unique solution, namely, $\theta\left(\phi_{\epsilon}\right)=\theta_{\epsilon}^{*}$ and $R\left(\phi_{\epsilon}\right)=\varepsilon$. Increases in $\phi$ only shift the destruction condition downward, increasing the equilibrium level of $R$ and decreasing the equilibrium level of $\theta$ (the creation condition is independent of $\phi$ ). In addition, for any given $\phi$, the creation condition (25) asymptotes the horizontal axis in $(R, \theta)$ space and the jobdestruction condition (24) grows without bound. Therefore $(a)-(d)$ follow for any $\phi>\phi_{\epsilon}$. Finally, $\phi_{\epsilon}-\varepsilon>0$ is equivalent to $\phi_{\epsilon}-R\left(\phi_{\epsilon}\right)>0$, which implies $(e)$.

\section{Proof of Proposition 2.}

Define $\Delta=\frac{(\varepsilon / R)^{\alpha} \tau(R)}{(r+\delta+\lambda) R}+\frac{\eta(\theta)}{\beta \theta q(\theta)}\left[1-\frac{\lambda(\varepsilon / R)^{\alpha}}{r+\delta+\lambda}\right]$. Since $\tau(R)>0$ by Proposition 1, it follows that $\Delta>0$ in any equilibrium. By totally differentiating (24) and (25),

$$
\begin{aligned}
\frac{\partial R}{\partial \tau_{e}} & =\frac{-\eta(\theta)}{\beta \theta q(\theta) \Delta}<0, \frac{\partial R}{\partial \tau_{f}}=-(1 / \Delta)\left[(\varepsilon / R)^{\alpha}+\frac{r \eta(\theta)}{\beta \theta q(\theta)}\right]<0, \\
\frac{\partial R}{\partial \tau_{h}} & =(1 / \Delta)(\varepsilon / R)^{\alpha}>0, \frac{\partial R}{\partial \tau_{b}}=-\frac{\partial R}{\partial \tau_{e}}>0
\end{aligned}
$$

and this concludes the proof.

Proof of Proposition 3. First, note that the corresponding distribution function is

$$
G(x)=\frac{\alpha \lambda(\alpha+\sigma) \varepsilon^{\alpha}}{\lambda(\alpha+\sigma)+\alpha \delta \varepsilon^{-\sigma}}\left\{\frac{\varepsilon^{-\alpha}}{\alpha}\left[1-\left(\frac{\varepsilon}{x}\right)^{\alpha}\right]+\frac{\delta \varepsilon^{-(\alpha+\sigma)}}{\lambda(\alpha+\sigma)}\left[1-\left(\frac{\varepsilon}{x}\right)^{\alpha+\sigma}\right]\right\}
$$

Verify that $G(\varepsilon)=0$ and $\lim _{x \rightarrow \infty} G(x)=1$. Under the parametric restrictions in the statement, $d G(x) \geq 0$ for all $x$, so $d G$ is a proper density. The restriction $\alpha>1$ ensures the mean is finite. 
Imposing steady states in (36), substituting (39) and solving for $H(x)$ reveals that $H(x)$ is as in $(21)$.

\section{Proof of Proposition 4.}

The problem is to find a cumulative distribution function $H$ that satisfies $H(R)=0$ and yields

$$
Y=a\left[\sigma_{1}\left(\hat{\kappa} K_{e}\right)^{\rho}+\sigma_{2} N^{\rho}\right]^{1 / \rho}
$$

where $\rho \in(0,1)$ and $a, \hat{\kappa}, \sigma_{1}$, and $\sigma_{2}$ are positive constants. Define $\varsigma(x)=\int_{x} z h(z) d z$ and $s(x)=1-H(x)$. Since, in general, $Y=\varsigma(\mu) K_{e}$ and $N=s(\mu) K_{e}$, (40) can be rewritten as $\varsigma(x)^{\rho}=a^{\rho}\left[\sigma_{1} \hat{\kappa}^{\rho}+\sigma_{2} s(x)^{\rho}\right]$. Differentiating both sides of this expression gives $\varsigma(x)=$ $\left(\frac{x}{\sigma_{2} a^{\rho}}\right)^{\frac{1}{1-\rho}} s(x)$. The last two equations yield $s(x)=\hat{\kappa}\left[\frac{1}{\sigma_{1}}\left(\frac{x}{\sigma_{2} a}\right)^{\frac{\rho}{1-\rho}}-\frac{\sigma_{2}}{\sigma_{1}}\right]^{-1 / \rho}$, which by defining

$$
\varepsilon=\sigma_{2} a\left(\sigma_{1}+\sigma_{2}\right)^{\frac{1-\rho}{\rho}} \text { and } \sigma=\frac{\sigma_{1}}{\sigma_{1}+\sigma_{2}}
$$

can be rewritten as $H(x)=1-\hat{\kappa}\left[\frac{1}{\sigma}\left(\frac{x}{\varepsilon}\right)^{\frac{\rho}{1-\rho}}-\frac{1-\sigma}{\sigma}\right]^{-1 / \rho}$. The requirement that $H(R)=0$ implies that $\hat{\kappa}=\kappa$ (with $\kappa$ as defined in Subsection 6.2). After specifying that $H(x)=0$ for $x<R$, this expression is identical to (38). So by construction, aggregation under (38) yields (40). And after letting $\hat{\kappa}=\kappa$ and making the substitutions in (41), one realizes that (40) is identical to the aggregate in Proposition 4. Finally, verifying that (38) is the truncation of (37) at $R$ concludes the proof.

To complete the analysis of Section 5, here I report the effects of all policies on market tightness:

$$
\begin{aligned}
\frac{\partial \theta}{\partial \tau_{e}} & =\frac{-(1-\beta) \theta q(\theta)(\varepsilon / R)^{\alpha} \tau(R)}{c \eta(\theta)(r+\delta+\lambda) R} \frac{\partial R}{\partial \tau_{e}}>0, \frac{\partial \theta}{\partial \tau_{h}}=\frac{1-\beta}{\beta c}\left[1-\frac{\lambda(\varepsilon / R)^{\alpha}}{r+\delta+\lambda}\right] \frac{\partial R}{\partial \tau_{h}}>0, \\
\frac{\partial \theta}{\partial \tau_{b}} & =\frac{-(1-\beta) \theta q(\theta)(\varepsilon / R)^{\alpha} \tau(R)}{c \eta(\theta)(r+\delta+\lambda) R} \frac{\partial R}{\partial \tau_{b}}<0, \frac{\partial \theta}{\partial \tau_{f}}=\frac{1-\beta}{\beta c}\left\{r+\left[1-\frac{\lambda(\varepsilon / R)^{\alpha}}{r+\delta+\lambda}\right] \frac{\partial R}{\partial \tau_{f}}\right\} .
\end{aligned}
$$

Without additional restrictions, the sign of $\partial \theta / \partial \tau_{f}$ is ambiguous. It is negative in any equilibrium with $\phi>\phi_{\epsilon}$ if $\delta>r(1-\varepsilon) / \varepsilon$. 


\section{B Appendix}

Lemma 1 Let $\varphi(R)=\int_{R}[1-G(x)] d x, \underline{\theta}=\frac{1-\beta}{\beta c}\left[\frac{\lambda}{r+\delta+\lambda} \varphi(0)-\phi-c\right]$. If $\tau_{b}$ is small and $q(\underline{\theta})>\frac{r+\delta+\lambda}{(1-\beta) \varphi(0)} c$, then there exists a unique pair $(\theta, R) \in \mathbb{R}_{+}^{2}$ that solves (15) and (16).

Proof. Differentiating (15) and (16), respectively, gives

$$
\begin{aligned}
\left.\frac{\partial R}{\partial \theta}\right|_{J D} & =\left\{\frac{(1-\beta)\left[1-(1-\beta) \tau_{b}\right]}{\beta c}\left[1-\frac{\lambda[1-G(R)]}{r+\delta+\lambda}-\frac{\tau_{b} \beta G^{\prime}(R) \varphi(R)}{\left[1-(1-\beta) \tau_{b}\right][1-G(R)]^{2}}\right]\right\}^{-1} \\
\left.\frac{\partial R}{\partial \theta}\right|_{J C} & =\frac{-(r+\delta+\lambda) c \eta(\theta)}{(1-\beta) \theta q(\theta)[1-G(R)]}
\end{aligned}
$$

where $\eta(\theta) \equiv-\theta q^{\prime}(\theta) / q(\theta)>0$. Notice that the job-creation condition is downward sloping in $(\theta, R)$ space, whereas the job-destruction condition is upward sloping, provided $\tau_{b}$ is not too big. To verify this, note that if $\tau_{b} \approx 0$, the slope of the job-destruction condition reduces to

$$
\left.\frac{\partial R}{\partial \theta}\right|_{J D}=\frac{\beta c}{(1-\beta)\left\{1-\frac{\lambda[1-G(R)]}{r+\delta+\lambda}\right\}}>0 .
$$

Therefore, as long as $\tau_{b}$ is small enough, if an intersection exists, it must be unique. To establish existence, define

$$
\begin{aligned}
T(R, \theta) & \equiv R-\frac{\tau_{b} \beta \tilde{x}(R)}{1-(1-\beta) \tau_{b}}-\frac{\left(1-\tau_{b}\right)(\phi+c)}{1-(1-\beta) \tau_{b}}-\frac{\beta c \theta}{(1-\beta)\left[1-(1-\beta) \tau_{b}\right]}+\frac{\lambda}{r+\delta+\lambda} \varphi(R) \\
\hat{T}(R, \theta) & \equiv \varphi(R)-\frac{(r+\delta+\lambda) c}{(1-\beta) q(\theta)} .
\end{aligned}
$$

The equilibrium conditions (15) and (16) are equivalent to $T(R, \theta)=0$ and $\hat{T}(R, \theta)=0$, respectively. Let $\underline{\theta}$ and $\bar{\theta}$ be defined by $T(0, \underline{\theta})=0$ and $\hat{T}(0, \bar{\theta})=0$, respectively. Since the $\theta$ that satisfies (16) goes to 0 as $R$ gets large, and the $\theta$ that satisfies (15) grows without bound as $R$ gets large, existence is guaranteed for parametrizations that satisfy $\underline{\theta}<\bar{\theta}$. Note that $\bar{\theta}$ solves $q(\bar{\theta})=\frac{r+\delta+\lambda}{(1-\beta) \varphi(0)} c$, and if $\tau_{b} \approx 0$, then $\underline{\theta}$ is as in the statement of the lemma. Thus, the condition $\underline{\theta}<\bar{\theta}$ is equivalent to the parametric restriction $q(\underline{\theta})>\frac{r+\delta+\lambda}{(1-\beta) \varphi(0)} c$. 


\section{Conditions for equilibrium labor hoarding.}

Since, as mentioned at the end of Section 6.2, having firm-worker pairs that sometimes choose to be inactive affects the shape of the aggregates in interesting ways, here I verify that it is indeed possible for the equilibrium of the basic model of Section 2 to exhibit this property.

With $G$ given by (20), (15) and (16) specialize to

$$
\begin{aligned}
{\left[1-\frac{\tau_{b} \beta \alpha}{(\alpha-1)\left[1-(1-\beta) \tau_{b}\right]}\right] R-\frac{\left(1-\tau_{b}\right)(\phi+c)}{1-(1-\beta) \tau_{b}}-\frac{\beta c \theta}{1-\beta}+\frac{\lambda \varepsilon^{\alpha} R^{1-\alpha}}{(\alpha-1)(r+\delta+\lambda)} } & =0 \\
\frac{\varepsilon^{\alpha} R^{1-\alpha}}{(\alpha-1)(r+\delta+\lambda)}-\frac{c}{q(\theta)(1-\beta)} & =0
\end{aligned}
$$

The following lemma provides conditions that ensure a unique equilibrium exists for this formulation.

Lemma 2 Let

$$
\underline{\theta}=\frac{(1-\beta)\left[1-(1-\beta) \tau_{b}\right]}{\beta c}\left\{\left[\left(1+\frac{\lambda}{(\alpha-1)(r+\delta+\lambda)}-\frac{\tau_{b} \alpha \beta}{(\alpha-1)\left[1-(1-\beta) \tau_{b}\right]}\right) \varepsilon-\frac{\left(1-\tau_{b}\right)(\phi+c)}{1-(1-\beta) \tau_{b}}\right]\right\} .
$$

Suppose $q(\underline{\theta})>\frac{(\alpha-1)(r+\delta+\lambda) c}{(1-\beta) \varepsilon}$ and $\tau_{b} \leq \bar{\tau}_{b}$, where

$$
\bar{\tau}_{b}=\frac{(\alpha-1)(r+\delta)}{\beta \alpha(r+\delta+\lambda)+(1-\beta)(\alpha-1)(r+\delta)} .
$$

Then there exists a unique pair $(\theta, R) \in(0, \infty) \times(\varepsilon, \infty)$ that satisfies (42) and (43).

Proof. Differentiating (42) and (43) gives

$$
\begin{aligned}
\left.\frac{\partial R}{\partial \theta}\right|_{J D} & =\left\{\frac{(1-\beta)\left[1-(1-\beta) \tau_{b}\right]}{\beta c}\left[1-\frac{\lambda(\varepsilon / R)^{\alpha}}{r+\delta+\lambda}-\frac{\tau_{b} \alpha \beta}{(\alpha-1)\left[1-(1-\beta) \tau_{b}\right]}\right]\right\}^{-1} \\
\left.\frac{\partial R}{\partial \theta}\right|_{J C} & =\frac{-(r+\delta+\lambda) c \eta(\theta)}{(1-\beta)(\varepsilon / R)^{\alpha} \theta q(\theta)},
\end{aligned}
$$

where $\eta(\theta) \equiv-\theta q^{\prime}(\theta) / q(\theta)>0$. Thus the job-creation condition (43) is always downward sloping in $(\theta, R)$ space, whereas the job-destruction condition (42) is upward sloping if $\tau_{b} \leq \bar{\tau}_{b}$. So under this condition, if an intersection exists, it is unique. The parameter restriction $q(\underline{\theta})>$ $\frac{(\alpha-1)(r+\delta+\lambda) c}{(1-\beta) \varepsilon}$ guarantees that the two lines intersect on $(0, \infty) \times(\varepsilon, \infty)$. 
Since the aim now is merely to show that hoarding may arise in equilibrium, I set $\tau_{b}=0$ to ease the algebra. ${ }^{46}$ Differentiating (42) and (43),

$$
\begin{aligned}
\frac{\partial R}{\partial \phi} & =\frac{(r+\delta+\lambda) \eta(\theta)}{\beta \theta q(\theta)[1-G(R)]+(r+\delta+\lambda) \eta(\theta)\left\{1-\frac{\lambda[1-G(R)]}{r+\delta+\lambda}\right\}}>0 \\
\frac{\partial \theta}{\partial \phi} & =\frac{-(1-\beta) \theta q(\theta)[1-G(R)]}{(r+\delta+\lambda) \eta(\theta) c} \frac{\partial R}{\partial \phi}<0,
\end{aligned}
$$

where $1-G(R)=(\varepsilon / R)^{\alpha}$ and $\eta(\theta) \equiv-\theta q^{\prime}(\theta) / q(\theta)$. An increase in $\phi$ has no direct effect on the job-creation condition, and it shifts the job-destruction condition upward in $\theta-R$ space. This increases the equilibrium value of $R$ and decreases the equilibrium value of $\theta$. Combining (42) and (43), one sees that the sign of $\phi-R$ is the sign of $\lambda / q(\theta)-[1-(1-\theta) \beta]$. So at low productivity realizations, the firm is more likely to hoard labor than to break the match when $\lambda$ is large (and hence the option value of keeping a match is large) and when $q$ is small (and hence the expected cost of hiring a new worker is high). Market tightness $\theta$ enters the expression with an ambiguous sign because on the one hand, a large $\theta$ makes hoarding more likely by increasing the expected recruiting cost; but on the other hand, through its effect on the worker's reservation wage, it also increases the value of her threat point in the wage bargain, which makes keeping an unproductive worker employed more costly and hoarding less likely. In fact, the latter effect disappears if the worker has no power in the wage bargain (i.e., if $\beta=0$ ). Next, I derive a condition on parameters that is sufficient for $R<\phi$ in equilibrium.

Let $\theta_{\varepsilon}^{*}$ be defined by $q\left(\theta_{\varepsilon}^{*}\right)=\frac{(\alpha-1)(r+\delta+\lambda) c}{(1-\beta) \varepsilon}$ and $\phi_{\varepsilon}=\left[1+\frac{\lambda}{(\alpha-1)(r+\delta+\lambda)}\right] \varepsilon-\left(1+\frac{\beta}{1-\beta} \theta_{\varepsilon}^{*}\right) c$. Then if $\phi=\phi_{\varepsilon}$, the versions of (42) and (43) with $\tau_{b}=0$ are solved by $\theta\left(\phi_{\varepsilon}\right)=\theta_{\varepsilon}^{*}$ and $R\left(\phi_{\varepsilon}\right)=\varepsilon$. Notice that if $R\left(\phi_{\varepsilon}\right)=\varepsilon<\phi_{\varepsilon}$, then there is a nondegenerate interval $\left[\phi_{\varepsilon}, \phi_{R}\right)$ such that $R(\phi)<\phi$ iff $\phi \in\left[\phi_{\varepsilon}, \phi_{R}\right)$. An example of the function $R(\phi)$ is illustrated in Figure 2 .

So a sufficient condition for the equilibrium to exhibit hoarding for at least some range of

\footnotetext{
${ }^{46}$ Just as for the existence and uniqueness results in Lemma 1 and Lemma 2 in Appendix B, the results that follow will also hold for replacement rates $\tau_{b}$ that are not too big. The algebra gets cumbersome with $\tau_{b}>0$ because under this specification, unemployment income $b$ is a function of the equilibrium distribution of wages earned.
} 


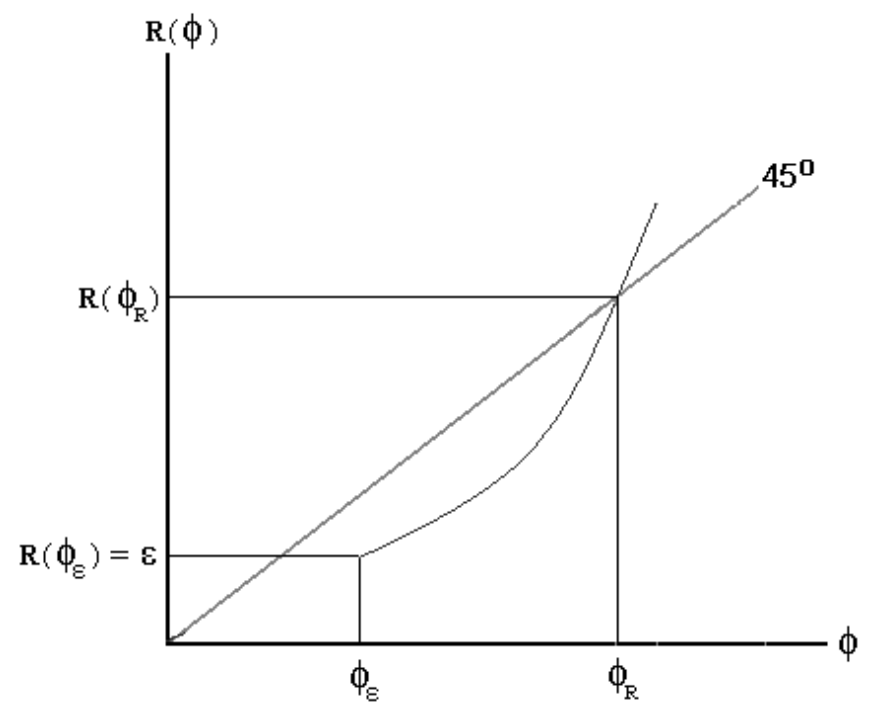

Figure 2: Destruction decision as a function of the variable cost

the parameter $\phi$ is that $\phi_{\varepsilon}-\varepsilon>0$, or equivalently, that $T(\lambda, \zeta)>0$, where

$$
T(\lambda, \zeta) \equiv \frac{\lambda \varepsilon}{(\alpha-1)(r+\delta+\lambda)}-\left(1+\frac{\beta}{1-\beta} \theta_{\varepsilon}^{*}\right) c
$$

The parameter $\zeta$ summarizes the efficiency of matching, with the property that $\partial m(u, v) / \partial \zeta>$ 0 and hence that $\partial q(\theta) / \partial \zeta>0$ for all $\theta$. Figure 3 plots the boundary $T(\lambda, \zeta)=0$ in $\lambda-\zeta$ space.

The condition $\phi_{\varepsilon}-\varepsilon>0$ is satisfied for the values of the parameters $\lambda$ and $\zeta$ that lie below the boundary. ${ }^{47}$ Intuitively, the parameter restriction that makes hoarding possible holds for relatively large $\lambda$ (i.e., when bad shocks are very transitory) and relatively low $\zeta$ (i.e., when the search process needed to replace the worker is very costly).

\footnotetext{
${ }^{47}$ Note that $\theta_{\varepsilon}^{*}$ goes to zero as $\zeta$ goes to zero. So $T(\lambda, 0)=0$ iff $\lambda=\lambda_{0}$, where $\lambda_{0} \equiv \frac{c(\alpha-1)(r+\delta)}{\varepsilon-c(a-1)}$ is the point at which the boundary intercepts the horizontal axis in Figure 3. Formally, this boundary is upward sloping because $\frac{\partial T}{\partial \zeta}=-\frac{\beta \theta_{\varepsilon}^{*}}{1-\beta} \frac{\partial \theta_{\varepsilon}^{*}}{\partial \zeta}<0$ and $\frac{\partial T}{\partial \lambda}=\frac{\varepsilon(r+\delta)}{(\alpha-1)(r+\delta+\lambda)^{2}}-\frac{b c^{2}(\alpha-1)}{(1-\beta) \varepsilon(1-\beta) q^{\prime}\left(\theta_{\varepsilon}^{*}\right)}>0$. The equilibrium may or may not exhibit hoarding for parametrizations that lie above the boundary.
} 


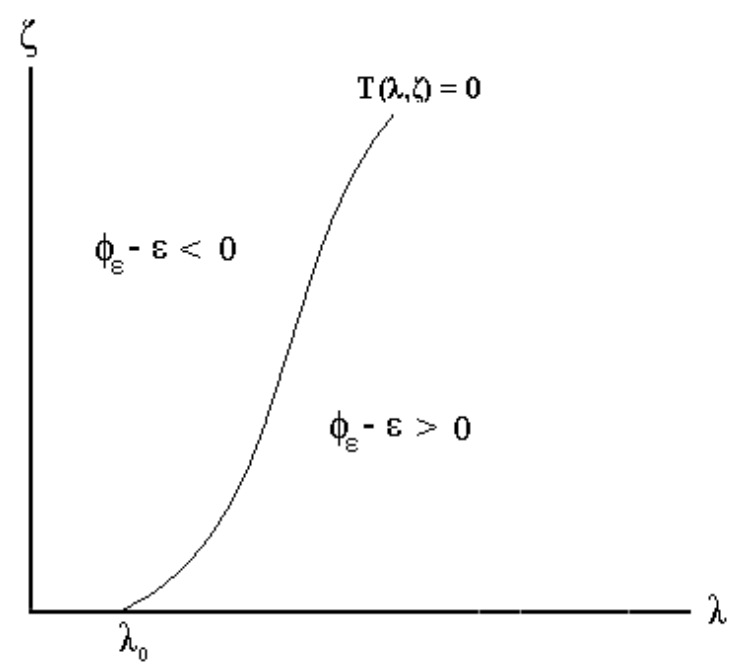

Figure 3: Range of parameters for which there is hoarding

\section{The model of Section 2 with a general fixed cost.}

The value functions are still given by $(4),(5),(6)$, and (7), but the profit function is now

$$
\pi(x)=x \min (n, k)-w(x)-c k-\phi n-C(x) k,
$$

where $C(x)$ is continuous and $C^{\prime}<0$ (the inequality need only be strict for $x<\phi$ ). The bargaining problem is unchanged, and hours are still given by (8), so

$$
\pi(x)=[\max (x-\phi, 0)-c-C(x)] k-w(x) .
$$

Also from the Nash bargaining, wages are

$$
w(x)=\beta[\max (x-\phi, 0)-c-C(x)] k+(1-\beta) r U,
$$

and therefore flow profits are

$$
\pi(x)=(1-\beta)\{[\max (x-\phi, 0)-c-C(x)] k-r U\} .
$$

Steps similar to the ones that led to (14) now imply

$$
S(x)=\frac{\max (x-\phi, 0)-\max (R-\phi, 0)+C(R)-C(x)}{r+\delta+\lambda} k .
$$


Note that if the idiosyncratic technology shock does not induce savings in the fixed cost (i.e., if either $C(x)=0$ for all $x$ or $\left.C^{\prime}=0\right)$, then (47) reduces to $(r+\delta+\lambda) S(x)=$ $[\max (x-\phi, 0)-\max (R-\phi, 0)] k$. From this it is clear that the expected surplus $S(x)$ is flat for $x<\phi$, meaning that generically, $R>\phi$ in an equilibrium with endogenous destruction. (The reservation $R$ is indeterminate in the interval $[0, \phi]$ in the knife-edge case in which the flat spot coincides with the horizontal axis.) Conversely, $C^{\prime}<0$ ensures that $S(x)$ is strictly increasing for all $x$, and therefore the level $R$ for which $S(R)=0$ may be to the right or to the left of $\phi$, depending on the parametrization. In general, $S(x)$ will have a kink at $x=\phi$, but this is immaterial for my purposes. The job-destruction condition for this case is

$$
p(R)-c-\frac{r U}{k}+\frac{\lambda}{r+\delta+\lambda} \hat{\varphi}(R)=0
$$

where $p(R) \equiv \max (R-\phi, 0)-C(R), r U$ is still given by (10), and

$$
\hat{\varphi}(R) \equiv \int_{R}[\max (x-\phi, 0)-\max (R-\phi, 0)+C(R)-C(x)] d G(x) .
$$

The job-creation condition is

$$
\hat{\varphi}(R)-\frac{(r+\delta+\lambda) c}{(1-\beta) q(\theta)}=0 .
$$

If one specifies the unemployment income by $b=\tau_{b} k$ as in Section 5 , it is immediate that (48) is increasing and (49) decreasing in $(\theta, R)$ space, so they will intercept exactly once under conditions analogous to those in Lemma 1. Alternatively, letting $b=\tau_{b} E_{G}[w(x) \mid x \geq R]$, (48) specializes to

$$
p(R)-\frac{\left(1-\tau_{b}\right) c}{1-(1-\beta) \tau_{b}}-\frac{\beta c \theta}{(1-\beta)\left[1-(1-\beta) \tau_{b}\right]}-\frac{\tau_{b} \beta \hat{E}(R)}{1-(1-\beta) \tau_{b}}+\frac{\lambda}{r+\delta+\lambda} \hat{\varphi}(R)=0
$$

where $\hat{E}(R) \equiv[1-G(R)]^{-1} \int_{R}[\max (x-\phi, 0)-C(x)] d G(x)$. For this formulation, conditions analogous to the ones in Lemma 2 (essentially a replacement rate $\tau_{b}$ that is not "too big") will guarantee existence and uniqueness of a pair $(\theta, R)$ that satisfies (49) and (50). Given the equilibrium $(\theta, R)$, the aggregation proceeds exactly as in Section 4 . 


\section{Reinterpreting the variable cost $\phi n$ as disutility from work.}

Instead of assuming there is a variable cost of production $\phi n$ as in Section 2, now suppose there is no such cost, but instead the worker suffers disutility $\phi n$ if she supplies $n$ hours to the firm. The value functions (4), (6), and (7) remain unchanged, whereas (5) is now

$$
r W(x)=w(x)-\phi n+\lambda \int \max [W(z)-U, 0] d G(z)-(\delta+\lambda)[W(x)-U] .
$$

The firm's flow profit is modified accordingly to $\pi(x)=x \min (n, k)-w(x)-c k-C(x) k^{48}$ The bargaining solution implies a choice of hours that is still characterized by (8). Naturally, regardless of which party bears the cost $\phi n$, the efficient bargaining solution will ensure the efficient number of hours worked and will use the wage $w(x)$ to distribute the surplus among the partners. In fact, since $W(x)$ is now given by (51), the first-order condition (9) now yields

$$
\begin{aligned}
& w(x)=\phi n+\beta[\max (x-\phi, 0)-c-C(x)] k+(1-\beta) r U \\
& \pi(x)=(1-\beta)\{[\max (x-\phi, 0)-c-C(x)] k-r U\}
\end{aligned}
$$

where $r U$ still satisfies (10). The outcome is that regardless of who suffers the production cost $\phi n$, the worker and firm share it so that the firm bears a fraction $1-\beta$. In fact, comparing (53) with (46) confirms that the firm's flow profit is unchanged when $\phi n$ is modeled in this way. Similarly, note that the worker's value function is also unchanged in this formulation relative to the variable cost interpretation. This can be verified by checking that substituting (52) into (51) delivers the same expression for the worker's value function as substituting (45) into (5).

\section{Derivation of the equilibrium conditions for the model with labor-market policies.}

In the model of Section 5, there are two reasons why the bargaining situation faced by a firm and a worker when they first meet and are still considering whether to form a match is different from the one they face every instant after having agreed to form the match. The first is that in the initial bargain, a one-time hiring subsidy is at stake. The second is that at that

\footnotetext{
${ }^{48}$ The fixed cost $C(x) k$ could be set to zero here. This, however, is of no consequence for the purposes of reinterpreting $\phi n$ as a disutility cost from work.
} 
point, the firm is not yet "locked in" by the firing tax. I use $w_{o}(x)$ to denote the wage that solves the initial bargain and $w(x)$ to denote the subsequent wage. Accordingly, $J_{o}(x)$ and $W_{o}(x)$ are the value functions for the firm and worker in the instant they form a new match and satisfy $W_{o}(x)-W(x)=J(x)-J_{o}(x)=w_{o}(x)-w(x)$.

The wages $w_{o}(x)$ and $w(x)$ are characterized by

$$
\beta\left[J_{o}(x)+\tau_{h} k\right]=(1-\beta)\left[W_{o}(x)-U\right] \quad \text { and } \quad \beta\left[J(x)+\tau_{f} k\right]=(1-\beta)[W(x)-U],
$$

where the equilibrium condition $V=0$ has already been imposed. Letting $S_{o}(x)=J_{o}(x)+$ $W_{o}(x)+\tau_{h} k-U$ and $S(x)=J(x)+W(x)+\tau_{f} k-U$ be the initial and the subsequent surpluses, respectively, the first-order conditions imply that $W_{o}(x)-U=\beta S_{o}(x), W(x)-U=\beta S(x)$, $J_{o}(x)+\tau_{h} k=(1-\beta) S_{o}(x)$, and $J(x)+\tau_{f} k=(1-\beta) S(x)$. Combining these with the value functions gives

$$
(r+\delta+\lambda) S(x)=(x-\phi-c) k+\tau_{e} k+r \tau_{f} k-r U+\lambda \int \max [S(z), 0] d G(z),
$$

with $r U$ as in (10). Since $S^{\prime}(x)>0$, there is a unique $R$ such that $S(x) \geq 0$ iff $x \geq R$. Using this reservation property, the surplus of an ongoing match can be written as

$$
(r+\delta+\lambda) S(x)=(x-\phi-c) k+\tau_{e} k+r \tau_{f} k-r U+\lambda \int_{R} S(z) d G(z),
$$

a natural generalization of (11). One can work with the value functions and the first-order conditions of the Nash problem to derive expressions for wages and profit. ${ }^{49}$ Evaluating (54) at $x=R$ implies

$$
\lambda \int_{R} S(z) d G(z)=r U-\left[(R-\phi-c) k+\tau_{e} k+r \tau_{f} k\right],
$$

and substituting this back into (54) yields (14). Using (14) to substitute $S(z)$ out of (54), evaluating at $x=R$ and using (10) produces the job-destruction condition:

$$
R-\phi-c+\tau_{e}+r \tau_{f}-\left(\tau_{b}+\frac{\beta}{1-\beta} c \theta\right)+\frac{\lambda}{r+\delta+\lambda} \int_{R}(x-R) d G(x)=0 .
$$

\footnotetext{
${ }^{49}$ The wages and profit in ongoing matches are given by $w(x)=\beta\left(x-\phi-c+\tau_{e}+r \tau_{f}\right) k+(1-\beta) r U$ and $\pi(x)=(1-\beta)[(x-\phi-c) k-r U]-\beta\left(\tau_{e}+r \tau_{f}\right) k$, whereas those agreed upon in an initial match are $w_{o}(x)=w(x)+\beta(r+\delta+\lambda)\left(\tau_{h}-\tau_{f}\right) k$ and $\pi_{o}(x)=\pi(x)-\beta(r+\delta+\lambda)\left(\tau_{h}-\tau_{f}\right) k$. All matches still set hours according to (8).
} 
Increases in the employment subsidy and the firing tax reduce $R$ for given $\theta$. In other words, an increase in $\tau_{e}$ or $\tau_{f}$ shifts the job-destruction condition downward in $\theta-R$ space. Conversely, an increase in $\tau_{b}$ raises the worker's outside option and hence increases $R$ for given $\theta$.

By free entry, $r V=0$, and hence

$$
(1-\beta) \int \max \left[S_{o}(x), 0\right] d G(x)=\frac{c k}{q(\theta)} .
$$

Note that $S_{o}(x)=S(x)+\left(\tau_{h}-\tau_{f}\right) k$, so in the presence of firing and hiring policies, the reservation strategy used for match formation, say $R_{o}$, may differ from $R$, the one used for match dissolution. However, there are several specifications for the hiring subsidy and the destruction tax under which $R_{o}=R$, and the analysis will focus on this subset of policies hereafter. ${ }^{50}$ Then, (55) becomes $\int_{R} S_{o}(x) d G(x)=\frac{c k}{(1-\beta) q(\theta)}$, which together with (14) yields the job-creation condition:

$$
\int_{R} \frac{x-R}{r+\delta+\lambda} d G(x)+[1-G(R)]\left(\tau_{h}-\tau_{f}\right)=\frac{c}{(1-\beta) q(\theta)} .
$$

With $G$ as in (20), the job-destruction and job-creation conditions specialize to (24) and (25).

\section{Steady-state distribution of productivities for the model of Section 7.}

Lemma 3 The steady-state distribution of productivities for the model in which initial productivity draws are from $G_{0}(x)=1-\left(\frac{\varepsilon_{0}}{x}\right)^{\alpha}$ if $\varepsilon_{0} \leq x$, and $G_{0}(x)=1-\left(\frac{\varepsilon_{0}}{x}\right)^{\alpha}=0$ otherwise, where $\varepsilon_{0}>0$ and subsequent draws are from (20), is given by (21).

Proof. Imposing steady states in the law of motion for the unknown distribution $H$ implies

$$
H(x)=\frac{1}{\delta+\lambda}\left\{\lambda+\left[\frac{\theta q(\theta) u}{1-u}\right]\left[\frac{G_{0}(x)-G_{0}(R)}{G(x)-G(R)}\right]\right\}[G(x)-G(R)],
$$

\footnotetext{
${ }^{50}$ The simplest example of one such policy is the self-financing policy that sets $\tau_{h}=\tau_{f}$ for all values of the idiosyncratic productivity draw $x$. But more generally, a policy specifying $\tau_{h}=\tau_{f}$ for all realizations $x \leq R$ and $\tau_{h}>\tau_{f}$ for all $x>R$ would also imply $R_{o}=R$. Note that a policy that merely specifies $\tau_{h}>\tau_{f}$ for all $x$ will be abused by firms and workers in the sense that since $R_{o}<R$, a firm-worker pair that meets and draws $x \in\left(R_{o}, R\right]$ will want to form a match only for an instant to collect $\left(\tau_{h}-\tau_{f}\right) k$ and destroy it right away (since right after collecting from the government, their break up decision is given by $R$ ). Yet another set of policies delivering $R_{o}=R$ is having $\tau_{f}>\tau_{h}=0$ but assuming $\tau_{f}$ is enforced from the very instant the firm-worker pair meet and start bargaining, which implies $w_{o}(x)=w(x)$, since in this case the firm is "locked in" by the firing tax upon meeting the worker.
} 
which generalizes the expression right above (2). In this model, (2) generalizes to

$$
u=\frac{\delta+\lambda G(R)}{\delta+\lambda G(R)+\theta q(\theta)\left[1-G_{0}(R)\right]},
$$

and using this to substitute $u /(1-u)$ from the expression for $H$ yields

$$
H(x)=\frac{\lambda\left[1-G_{0}(R)\right][G(x)-G(R)]+[\delta+\lambda G(R)]\left[G_{0}(x)-G_{0}(R)\right]}{(\delta+\lambda)\left[1-G_{0}(R)\right][G(x)-G(R)]}[G(x)-G(R)] .
$$

Substituting the parametric expressions for $G_{0}$ and $G$ in the first factor reveals that it equals $(\varepsilon / R)^{-\alpha}$, but this is just $[1-G(R)]^{-1}$, so (56) can be written as (3) or, given the Pareto assumption, as (21).

\section{Sensitivity of the quantitative results.}

Table 2 reports results for $\alpha=1.1$, a value that implies the distribution of output in the model is close to satisfying Zipf's law. For this case, the values of the calibrated parameters are $\lambda=1.2, \phi=1.13, \varepsilon_{0}=0.34$, and $\tau_{b}=1.15$. In the equilibrium, $R=1.08$. The means of the distributions $G$ and $G_{0}$ are 11 and 3.73, respectively. The average wage is $\bar{w}=10.47$. The responses to policy are extremely large. For instance, an increase in unemployment benefits of one-tenth of $1 \%$ increases TFP as measured by $A$ by $4.5 \%$, and decreases it by $30 \%$ according to $\hat{A}$ and by $32 \%$ according to $\tilde{A}$.

\begin{tabular}{lrrrrcccc}
\hline \hline \multicolumn{2}{l}{} & $\tau_{i} / \bar{w}$ & \multicolumn{1}{c}{$D$} & $u$ & $A_{\tau_{i}} / A_{0}$ & $\hat{A}_{\tau_{i}} / \hat{A}_{0}$ & $\tilde{A}_{\tau_{i}} / \tilde{A}_{0}$ & $H(\phi)$ \\
\hline \multicolumn{2}{l}{ baseline } & 0.110 & 10 & 0.069 & 1 & 1 & 1 & 0.050 \\
\hline$\tau_{b}$ & 1.210 & 0.111 & 7 & 0.106 & 1.045 & 0.696 & 0.674 & 0.003 \\
& 1.109 & 0.109 & 17 & 0.040 & 0.967 & 1.585 & 1.655 & 0.085 \\
\hline$\tau_{f}$ & 0.015 & 0.001 & 12 & 0.055 & 0.984 & 1.210 & 1.231 & 0.067 \\
& 0.025 & 0.002 & 16 & 0.045 & 0.973 & 1.425 & 1.473 & 0.078 \\
\hline \multirow{2}{*}{$\tau_{e}$} & 0.015 & 0.001 & 12 & 0.059 & 0.988 & 1.141 & 1.155 & 0.062 \\
& 0.025 & 0.002 & 13 & 0.052 & 0.981 & 1.263 & 1.291 & 0.070 \\
\hline \multirow{2}{*}{$\tau_{h}$} & 0.015 & 0.001 & 8 & 0.082 & 1.015 & 0.863 & 0.852 & 0.034 \\
& 0.025 & 0.002 & 7 & 0.090 & 1.025 & 0.795 & 0.778 & 0.023 \\
\hline
\end{tabular}

Table 2: Parametrization with $\alpha=1.1$

Table 3 reports results for $\alpha=3$. The values of the calibrated parameters are $\lambda=1.28$, $\phi=1.045, \varepsilon_{0}=0.9$, and $\tau_{b}=0.05$. In the equilibrium, $R=1.027$. The means of the 
distributions $G$ and $G_{0}$ are 1.5 and 1.34 , respectively. The average wage is $\bar{w}=0.47$. The effects are now smaller than those in Table 1. For example, increasing the replacement rate from $11 \%$ to $12 \%$ increases TFP as measured by $A$ by only half a percentage point. With TFP measured by $\hat{A}$ and $\tilde{A}$, this policy implies a reduction of $2.4 \%$ and $6 \%$, respectively.

\begin{tabular}{lccrccccc}
\hline \hline \multicolumn{2}{l}{} & $\tau_{i} / \bar{w}$ & \multicolumn{1}{c}{$D$} & $u$ & $A_{\tau_{i}} / A_{0}$ & $\hat{A}_{\tau_{i}} / \hat{A}_{0}$ & $\tilde{A}_{\tau_{i}} / \tilde{A}_{0}$ & $H(\phi)$ \\
\hline \multicolumn{2}{l}{ baseline } & 0.11 & 10 & 0.069 & 1 & 1 & 1 & 0.050 \\
\hline$\tau_{b}$ & 0.058 & 0.12 & 8 & 0.082 & 1.005 & 0.976 & 0.940 & 0.035 \\
& 0.049 & 0.10 & 11 & 0.063 & 0.997 & 1.012 & 1.033 & 0.057 \\
\hline$\tau_{f}$ & 0.005 & 0.01 & 13 & 0.054 & 0.994 & 1.033 & 1.091 & 0.067 \\
& 0.010 & 0.02 & 18 & 0.039 & 0.988 & 1.073 & 1.211 & 0.084 \\
\hline \multirow{2}{*}{$\tau_{e}$} & 0.005 & 0.01 & 12 & 0.058 & 0.996 & 1.022 & 1.059 & 0.062 \\
& 0.010 & 0.02 & 14 & 0.047 & 0.991 & 1.047 & 1.130 & 0.074 \\
\hline \multirow{2}{*}{$\tau_{h}$} & 0.005 & 0.01 & 8 & 0.083 & 1.006 & 0.972 & 0.929 & 0.034 \\
& 0.010 & 0.02 & 7 & 0.096 & 1.011 & 0.949 & 0.871 & 0.018 \\
\hline
\end{tabular}

Table 3: Parametrization with $\alpha=3$

Table 4 reports results for an alternative calibration strategy, namely keeping $\eta=0.72$ but calibrating $\beta$ jointly with $\left(\lambda, \varepsilon_{0}, \tau_{b}, \phi\right)$ so that the model also reproduces a labor share of 0.66 when all of the firm profit is imputed as capital income. The value of $\alpha$ is set to 1.15. The values of the calibrated parameters are $\beta=0.1, \lambda=0.255, \phi=1.611, \varepsilon_{0}=0.267$, and $\tau_{b}=0.6947$. In the equilibrium, $R=1.541$. The means of the distributions $G$ and $G_{0}$ are 7.67 and 2.05, respectively. The average wage is $\bar{w}=6.316$.

\begin{tabular}{lccrccccc}
\hline \hline & & $\tau_{i} / \bar{w}$ & \multicolumn{1}{c}{$D$} & $u$ & $A_{\tau_{i}} / A_{0}$ & $\hat{A}_{\tau_{i}} / \hat{A}_{0}$ & $\tilde{A}_{\tau_{i}} / \tilde{A}_{0}$ & $H(\phi)$ \\
\hline \multicolumn{2}{l}{ baseline } & 0.11 & 10 & 0.069 & 1 & 1 & 1 & 0.050 \\
\hline$\tau_{b}$ & 0.770 & 0.12 & 9 & 0.077 & 1.044 & 0.945 & 0.938 & 0.002 \\
& 0.630 & 0.10 & 11 & 0.062 & 0.963 & 1.059 & 1.067 & 0.091 \\
\hline \multirow{2}{*}{$\tau_{f}$} & 0.650 & 0.10 & 12 & 0.053 & 0.918 & 1.170 & 1.195 & 0.139 \\
& 1.250 & 0.20 & 16 & 0.035 & 0.824 & 1.543 & 1.639 & 0.239 \\
\hline$\tau_{e}$ & 0.075 & 0.01 & 11 & 0.061 & 0.957 & 1.069 & 1.079 & 0.097 \\
& 0.320 & 0.05 & 17 & 0.033 & 0.818 & 1.522 & 1.613 & 0.246 \\
\hline \multirow{2}{*}{$\tau_{h}$} & 0.075 & 0.01 & 10 & 0.070 & 1.008 & 0.987 & 0.985 & 0.041 \\
& 0.320 & 0.05 & 9 & 0.075 & 1.033 & 0.950 & 0.944 & 0.013 \\
\hline
\end{tabular}

Table 4: Alternative calibration strategy 
A $1 \%$ increase in the replacement ratio increases TFP as measured by $A$ by $4.4 \%$. Introducing firing taxes equivalent to $10 \%$ of the average wage reduces $A$ by $8.9 \%$. This policy also reduces the unemployment rate and increases the labor share to 0.71. (It increases the labor share because the lower unemployment rate puts the worker in a stronger bargaining position vis-à-vis the firm.) According to $\hat{A}$ and $\tilde{A}$, this firing tax increases TFP by $17 \%$ and $19.5 \%$, respectively. A $1 \%$ employment subsidy reduces TFP as measured by $A$ by $4.3 \%$, but increases it according to $\hat{A}$ and $\tilde{A}$, by $6.9 \%$ and $7.9 \%$, respectively. The policy reduces unemployment, which increases the labor share to 0.696 . 


\section{References}

[1] Acemoglu, Daron, and Fabrizio Zilibotti. "Productivity Differences." Quarterly Journal of Economics 116(2) (May 2001): 563-606.

[2] Axtell, Robert L. "Zipf Distribution of U.S. Firm Sizes." Science 293 (September 2001): $1818-1820$.

[3] Blanchard, Olivier J. "The Medium Run." Brookings Papers on Economic Activity 1997(2): $89-158$.

[4] Burnside, Craig, Martin Eichenbaum, and Sergio Rebelo. "Labor Hoarding and the Business Cycle." Journal of Political Economy 101(2) (April 1993): 245-273.

[5] Chari, V. V., Diego Restuccia, and Carlos Urrutia. "On-the-Job Training, Limited Commitment, and Firing Costs." University of Minnesota, mimeo, 2005.

[6] Cobb, Charles W., and Paul H. Douglas. "A Theory of Production." American Economic Review 18(1) (March 1928): 138-165.

[7] Conlisk, John. "An Aggregate Model of Technical Change." Quarterly Journal of Economics 104(4) (November 1989): 787-821.

[8] Fisher, Franklin M. "The Existence of Aggregate Production Functions." Econometrica 37(4) (October 1969): 553-577.

[9] Gollin, Douglas. "Getting Income Shares Right." Journal of Political Economy 110(2) (April 2002): 458-474.

[10] Hagedorn, Marcus, and Iourii Manovskii. "The Cyclical Behavior of Equilibrium Unemployment and Vacancies Revisited." University of Pennsylvania, mimeo, 2005. 
[11] Hall, Robert E., and Charles I. Jones. "Why Do Some Countries Produce So Much More Output Per Worker Than Others?" Quarterly Journal of Economics 114(1) (February 1999): 83-116.

[12] Hopenhayn, Hugo, and Richard Rogerson. "Job Turnover and Policy Evaluation: A General Equilibrium Analysis." Journal of Political Economy 101(5) (October 1993): 915-938.

[13] Houthakker, Hendrik S. "The Pareto Distribution and the Cobb-Douglas Production Function in Activity Analysis." Review of Economic Studies 23(1) (1955-1956): 27-31.

[14] Klenow, Peter J., and Andrés Rodríguez-Clare. "The Neoclassical Revival in Growth Economics: Has It Gone Too Far?" NBER Macroeconomics Annual 1997, edited by Ben S. Bernanke and Julio J. Rotemberg. Cambridge: MIT Press, 1997.

[15] Levhari, David. "A Note on Houthakker's Aggregate Production Function in a Multifirm Industry." Econometrica 36(1) (January 1968): 151-154.

[16] Ljungqvist, Lars, and Thomas J. Sargent. Recursive Macroeconomic Theory. Cambridge: MIT Press, 2000.

[17] Marimon, Ramon, and Fabrizio Zilibotti. "Unemployment vs. Mismatch of Talents: Reconsidering Unemployment Benefits." Economic Journal 109 (April 1999): 266-291.

[18] Millard, Stephen P., and Dale T. Mortensen. "The Unemployment and Welfare Effects of Labour Market Policy: A Comparison of the USA and the UK." In Unemployment Policy: Government Options for the Labour Market, edited by Dennis J. Snower and Guillermo de la Dehesa. Cambridge: Cambridge University Press, 1997.

[19] Mortensen, Dale T., and Christopher A. Pissarides. "Job Creation and Job Destruction in the Theory of Unemployment." Review of Economic Studies 61(3) (1994): 397-415.

[20] OECD. "The OECD Jobs Study: Facts, Analysis, Strategies" (1994). Organisation for Economic Co-operation and Development. 
[21] Parente, Stephen L., and Edward C. Prescott. "Barriers to Technology Adoption and Development." Journal of Political Economy 102(2) (April 1994): 298-321.

[22] Parente, Stephen L., and Edward C. Prescott. Barriers to Riches. Cambridge: MIT Press, 2000.

[23] Petrongolo, Barbara, and Christopher A. Pissarides. "Looking into the Black Box: A Survey of the Matching Function." Journal of Economic Literature 39(2) (June 2001): $390-431$.

[24] Pissarides, Christopher A. Equilibrium Unemployment Theory. 2nd ed. Cambridge: MIT Press, 2000.

[25] Prescott, Edward C. "Lawrence R. Klein Lecture 1997: Needed: A Theory of Total Factor Productivity." International Economic Review 39(3) (August 1998): 525-551.

[26] Restuccia, Diego, and Richard Rogerson. "Policy Distortions and Aggregate Productivity with Heterogeneous Plants." Arizona State University, mimeo, 2004.

[27] Shimer, Robert. "The Cyclical Behavior of Equilibrium Unemployment and Vacancies." American Economic Review 95(1) (March 2005): 25-49.

[28] Veracierto, Marcelo. "Employment Flows, Capital Mobility, and Policy Analysis." International Economic Review 42(3) (August 2001): 571-596. 